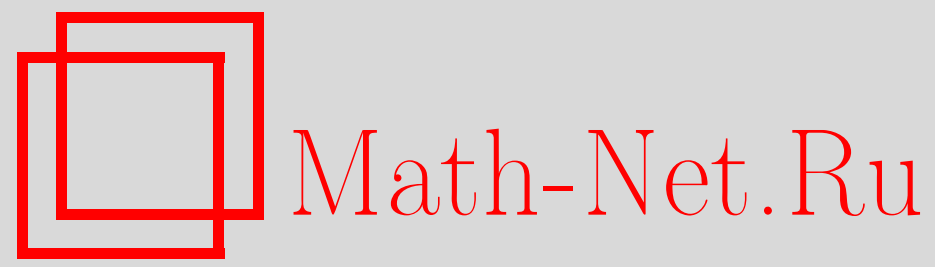

А. А. Болибрух, Дифференциальные уравнения с мероморфными коэффициентами, Совр. пробл. матем., 2003, выпуск 1, 29-82

DOI: https://doi.org/10.4213/spm3

Использование Общероссийского математического портала MathNet.Ru подразумевает, что вы прочитали и согласны с пользовательским соглашением

http://www . mathnet.ru/rus/agreement

Параметры загрузки:

IP: 35.173 .219 .12

26 апреля 2023 г., $17: 23: 20$

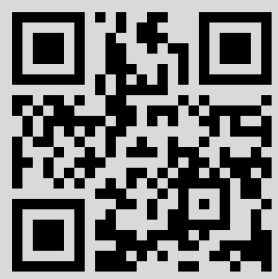




\title{
Дифференциальные уравнения с мероморфными коэффициентами
}

\author{
А. А. Болибрух
}

\section{Введение}

В настоящей работе рассматриваются следующие задачи аналитической теории дифференциальных уравнений: 21-я проблема Гильберта для фуксовых систем линейных дифференциальных уравнений, проблема нормальной формы Биркгофа для системы линейных дифференциальных уравнений с иррегулярной особой точкой и проблема классификации изомонодромных деформаций фуксовых систем.

Во всех трех случаях речь идет о дифференциальных уравнениях с мероморфными коэффициентами, линейных в первых двух упомянутых проблемах и нелинейных уравнениях в частных производных в третьей проблеме. В настоящей работе показано, что все три эти проблемы, занимающие центральное место в аналитической теории дифференциальных уравнений, тесно связаны между собой и что методы решения одной из них могут быть с успехом применены к решению других.

Главным результатом представляемой работы является решение 21-й проблемы Гильберта для линейных фуксовых систем.

Эта проблема связана с некоторым специальным классом линейных дифференциальных уравнений на сфере Римана $\overline{\mathbb{C}}$, с классом так называемых фуксовых систем.

Рассмотрим систему линейных дифференциальных уравнений

$$
d f=\omega f
$$

(c) Болиьрух А. А., 2003 
на сфере Римана, где $\omega=\left\|\omega_{i j}\right\|, 1 \leqslant i, \underline{j} \leqslant p,-$ матрица дифференциальных 1-форм, голоморфных на $\overline{\mathbb{C}}$ за исключением некоторого конечного множества $D$ особых точек $\left\{a_{1}, \ldots, a_{n}\right\}$. Точка $a_{i}$ называется фуксовой точкой системы $(0.1)$, если форма $\omega$ имеет в этой точке полюс первого порядка. Обозначим через $B^{i}=\operatorname{res}_{a_{i}} \omega$ вычеты формы $\omega$ в точках $a_{i}$. Если точки $\infty$ нет среди особых точек фуксовой системы (0.1), то по теореме о сумме вычетов

$$
\sum_{i=1}^{n} B^{i}=0 .
$$

Фуксова система в координате $z$ комплексной плоскости может быть записана следующим образом:

$$
\frac{d f}{d z}=\left(\sum_{i=1}^{n} B^{i} \frac{1}{z-a_{i}}\right) f .
$$

Рассмотрим в окрестности точки $z_{0} \in \overline{\mathbb{C}} \backslash D$ фундаментальную матрицу $Y(z)$ пространства $X$ решений системы $(0.1)$ (столбцы матрицы $Y(z)$ образуют базис в $X)$. Обозначим через $Y^{\prime}(z)$ аналитическое продолжение $Y(z)$ вдоль петли $\gamma$, лежащей в $\overline{\mathbb{C}} \backslash D$. Матрица $Y^{\prime}(z)$ также является фундаментальной матрицей пространства $X$, поэтому

$$
Y^{\prime}(z) G=Y(z), \quad G \in G L(p ; \mathbb{C}) .
$$

Сопоставление $\gamma \mapsto G$ зависит лишь от гомотопического класса $[\gamma]$ петли $\gamma$ и задает гомоморфизм

$$
\chi: \pi_{1}\left(\overline{\mathbb{C}} \backslash D, z_{0}\right) \rightarrow G L(p ; \mathbb{C})
$$

фундаментальной группы пространства $\overline{\mathbb{C}} \backslash D$ в группу невырожденных комплексных матриц порядка $p$. Гомоморфизм (0.4) называется представлением монодромии или просто монодромией системы (0.1), а группа $\operatorname{Im} \chi$ - группой монодромии этой системы. При замене точки $z_{0}$ на $z_{0}^{\prime}$ или при замене фундаментальной матрицы $Y(z)$ матрицы монодромии $G$ переходят в $S^{-1} G S$, где $S$ - неособая постоянная матрица. Тем самым монодромия системы (0.1) определена с точностью до эквивалентности. 
Аналогично определяется представление монодромии и для скалярных линейных дифференциальных уравнений с мероморфными коэффициентами

$$
u^{(p)}+q_{1}(z) u^{(p-1)}+\cdots+q_{p}(z) u=0 .
$$

При этом вместо матрицы $Y(z)$ следует рассмотреть матрицу, $i$-й столбец которой имеет вид $\left(u_{i}, \frac{d u_{i}}{d z} \ldots \frac{d^{p-1} u_{i}}{d z^{p-1}}\right)^{t}$, где $u_{1}, \ldots, u_{p}-$ базис в пространстве решений уравнения (0.5), а $t$ означает транспонирование. Уравнение (0.5) называется фуксовым в особой точке $a_{i}$, если порядок полюса коэффициента $q_{j}(z)$ этого уравнения в точке $a_{i}$ не превосходит числа $j$.

Все особые точки являются для фуксовой системы (0.3) регулярными особыми точками. Последнее означает, что любое решение $f$ при приближении к особой точке $a_{i}$ по любой секториальной окрестности с вершиной в точке $a_{i}$, не совпадающей с $\mathbb{C}$, растет не быстрее некоторой степени расстояния $\left|z-a_{i}\right|$ до этой точки.

Класс систем (0.1) с регулярными особыми точками содержит в себе класс фуксовых систем, но не исчерпывается им ${ }^{1}$.

В отличие от систем, для уравнений (0.5) понятия фуксовости и регулярности эквивалентны ${ }^{2}$.

2. Задача восстановления фуксова уравнения по его монодромии (0.4) впервые упоминается Риманом в одной из заметок конца 1850-х годов ${ }^{3}$. В 1900 г. она была включена Д. Гильбертом в число его "Математических проблем" под номером XXI и сформулирована следующим образом ${ }^{4}$ :

"Показать, что всегда существует линейное дифференциальное уравнение фуксова типа с заданными особыми точками и заданной группой монодромии".

Сложилась традиция, по которой эта проблема применительно к фуксовым системам называется в литературе проблемой Римана-Гильберта. (Аналогичное название употребляется и для

\footnotetext{
${ }^{1}$ Хартман Ф. Обыкновенные дифференциальные уравнения. М.: Мир, 1970.

${ }^{2}$ Ibid.

${ }^{3}$ Риман Б. Сочинения. М.: Гостехтеоретиздат, 1948.

${ }^{4}$ Проблемы Гильберта. М.: Наука, 1969.
} 
другой задачи, которая здесь не рассматривается ${ }^{5}$.) Конформным преобразованием $\overline{\mathbb{C}}$ всегда можно добиться того, чтобы точки $\infty$ не было среди особых точек системы (0.1), поэтому проблему Римана-Гильберта можно сформулировать так:

Дано представление (0.4). Показать, что всегда существует система (0.3), (0.2) с заданной монодромией (0.4).

Долгое время считалось, что проблема Римана-Гильберта полностью решена И. Племелем в работе 1908 года ${ }^{6}$. Однако в начале 1980-х годов в его доказательстве были обнаружены лакуны ${ }^{7}$. Метод решения, предложенный Племелем, состоял в сведении проблемы Римана-Гильберта к так называемой однородной граничной задаче Гильберта теории сингулярных интегральных уравнений. Подробное исследование последней задачи содержится в книге Н.И. Мусхелишвили ${ }^{8}$ и уже упомянутой книге Н. П. Векуа. Указанное сведение осуществляется следующим образом.

Пусть все точки $a_{1}, \ldots, a_{n}$ лежат в конечной комплексной плоскости. Соединим их простым замкнутым контуром $L$ (см. диаграмму).

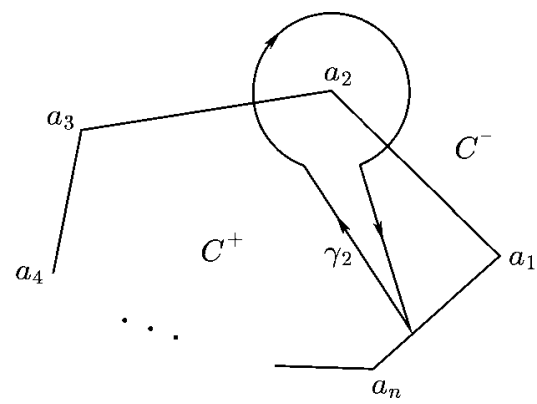

Зададим на $L$ кусочно постоянную невырожденную матричную функцию $g(t)$ следующим образом:

$$
g(t)=G_{i} \cdots G_{1}, \quad t \in\left[a_{i} ; a_{i+1}\right),
$$

\footnotetext{
${ }^{5}$ См., например, Векуа Н.П. Системы сингулярных интегральных уравнений и некоторые граничные задачи. М.: Наука, 1970.

${ }^{6}$ Plemelj J. Problems in the sense of Riemann and Klein. New York: Interscience Publ., 1964.

${ }^{7}$ Арнолъд В.И., Ильяшенко Ю.С. Обыкновенные дифференциальные уравнения // Динамические системы I. Итоги науки и техники. Соврем. проблемы матем. Фундам. напр. Т. 1. М.: ВИНИТИ, 1985; Treibich Kohn A. Un résultat de Plemelj // Progr. Math., 1983, 37, 307-312.

${ }^{8}$ Мусхелишвили Н. И. Сингулярные интегральные уравнения. М.: Наука, 1968.
} 
где $G_{i}$ - матрица монодромии $(0.4)$, соответствующая обходу точки $a_{i}$ по "малой" петле. Обозначим через $\mathbb{C}^{+}$область конечной плоскости комплексного переменного, ограниченную контуром $L$, через $\mathbb{C}^{-}$- дополнение к $\mathbb{C}^{+}$в $\overline{\mathbb{C}}$.

Рассмотрим следующую задачу: найти все такие пары векторфункций $\varphi^{+}=\left(\varphi_{(1)}^{+}, \ldots, \varphi_{(p)}^{+}\right)$и $\varphi^{-}=\left(\varphi_{(1)}^{-}, \ldots, \varphi_{(p)}^{-}\right)$, что:

1) $\varphi^{+}(z)$ голоморфна в $\mathbb{C}^{+}, \varphi^{-}(z)$ голоморфна в $\mathbb{C}^{-}$и имеет степенной рост в точке $\infty$;

2) $\varphi^{+}(z), \varphi^{-}(z)$ непрерывны вплоть до контура $L$ за исключением точек $a_{1}, \ldots, a_{n}$ и на $\left(a_{i}, a_{i+1}\right)$ связаны соотношением

$$
\varphi^{+}(t) g(t)=\varphi^{-}(t)
$$

3) $\varphi^{ \pm}(z)\left(z-a_{i}\right)^{\varepsilon}$ стремится к нулю для некоторого $0 \leqslant \varepsilon<1$ при приближении точки $z \mathrm{k} a_{i}$ по областям $\mathbb{C}^{+}$и $\mathbb{C}^{-}$соответственно.

Эта задача сводится к задаче с непрерывной функцией $g(t)$ (Племель и Векуа осуществляют такое сведение по-разному), которая затем решается методами теории сингулярных интегральных уравнений. При этом оказывается, что всегда существует такая система решений $\varphi_{1}^{ \pm}(z), \ldots, \varphi_{p}^{ \pm}(z)$, для которой выполнены следующие условия:

а) определитель матрицы $Y$, строчки которой составлены из вектор-функций $\varphi_{1}^{ \pm}(z), \ldots, \varphi_{p}^{ \pm}(z)$, отличен от нуля во всех точках комплексной плоскости $\mathbb{C}$ за исключением точек из $D$;

б) матрица $z^{S} \cdot Y(z)$, где $S$ - некоторая целочисленная диагональная матрица, голоморфно обратима в точке $\infty$.

Функция $\varphi_{l}^{+}(z)$ из этой системы решений допускает аналитическое продолжение вдоль любого пути, не пересекающего множества $D$ особых точек, в любую точку $\mathbb{C} \backslash D$. Из свойства 2) следует, что при продолжении $\varphi_{l}^{+}$вдоль "малой" петли, обходящей точку $a_{i}, \varphi_{l}^{+}$переходит в $\varphi_{l}^{+} G_{i}^{-1}$ (на диаграмме $\left.i=2\right)$, поэтому то же самое верно и для матрицы $Y(z)$. Таким образом, при аналитическом продолжении вдоль петли $\gamma$ с началом и концом в точке $z_{0}$ матрица $Y$ переходит в $\widetilde{Y}$, где

$$
\tilde{Y} G=Y, \quad G=\chi([\gamma]) .
$$

То же самое верно и для матрицы

$$
Y^{\prime}=\left(z-a_{1}\right)^{S} Y,
$$


А. А. Болибрух

которая уже является голоморфно обратимой в точке $\infty$ согласно свойству б) построенной системы решений.

Отсюда и из свойства а) следует, что матричная форма

$$
\omega=d Y^{\prime}\left(Y^{\prime}\right)^{-1}
$$

однозначна на $\overline{\mathbb{C}}$ и голоморфна вне особых точек $a_{1}, \ldots, a_{n}$ из $D$. Система (0.1) с матричной формой (0.6) имеет заданную монодромию (0.4), а точки $a_{1}, \ldots, a_{n}$ являются для нее регулярными особыми точками.

Далее Племель применяет некоторую процедуру, с помощью которой переходит от построенной системы к другой с той же монодромией и с теми же особыми точками, которая уже является фуксовой во всех точках, кроме, быть может, одной. Доказательство Племеля в этой части не вызывает никаких возражений. Что же касается утверждения Племеля о том, что в последней точке систему также можно привести к фуксовой, то строгое доказательство в общем случае в его работе отсутствует. Однако рассуждение Племеля можно довести до конца, если одна из матриц монодромии $G_{i}$ диагонализируема ${ }^{9}$.

Итак, в работе Племеля доказана разрешимость проблемы Римана-Гильберта в случае, когда одна из матриц монодромии $G_{i}$, соответствующая обходу точки $a_{i}$ по "малой" петле, диагонализируема.

Племель также первым решил проблему, аналогичную проблеме Римана-Гильберта, в классе систем с регулярными особыми точками.

После опубликования результата тематика работ, связанных с проблемой Римана-Гильберта, переместилась в основном в область эффективного построения матриц фуксовой системы по заданным матрицам монодромии $G_{1}, \ldots, G_{n}$. В конце 1920 -х годов И. А. Лаппо-Данилевский с помощью развитого им метода аналитических функций от матриц представил решения фуксовой системы и матрицы монодромии $G_{1}, \ldots, G_{n}$ в виде сходящихся рядов от матриц коэффициентов этой системы ${ }^{10}$. Эффективное

${ }^{9}$ Арнолъд В.И., Илъяшенко Ю. С. Обыкновенные дифференциальные уравнения // Динамические системы I. Итоги науки и техники. Соврем. проблемы матем. Фундам. напр. Т. 1. М.: ВИНИТИ, 1985.

10 Лаппо-Данилевский И. А. Применение функций от матриц к теории линейных систем обыкновенных дифференциальных уравнений. М.: Гостехтеоретиздат, 1957. 
решение проблемы Римана-Гильберта сводилось в этом случае к обращению полученных рядов и исследованию вопроса сходимости, который был решен в его работе положительно для матриц $G_{1}, \ldots, G_{n}$, близких к единичной матрице.

Тем самым Лаппо-Данилевский доказал разрешимость проблемы Римана-Гильберта для представлений (0.4), матрицы монодромии которых, соответствующие обходам точек $a_{i}$ по "малым" петлям, близки к единичной матрице.

В 1956 г. разрешимость проблемы Римана-Гильберта для представления (0.4) размерности $p=2$ в случае трех особых точек доказал Б.Л. Крылов, построив эффективное решение задачи ${ }^{11}$.

Аналогичную задачу для четырех особых точек рассмотрел Н. П. Еругин ${ }^{12}$.

Новый этап в изучении проблемы Римана-Гильберта открыла работа Х. Рёрля ${ }^{13} 1957$ года, впервые применившего к ее решению методы теории расслоений. (Фактически соображения такого рода восходят к Г. Биркгофу ${ }^{14}$, передоказавшему результат Племеля, однако в то время адекватного геометрического языка для соответствующего описания не было.)

По представлению (0.4) Рёрль строит главное расслоение $F^{\prime}$ на $\overline{\mathbb{C}} \backslash D$ со структурной группой $G L(p ; \mathbb{C})$. Построенное расслоение оказывается голоморфно тривиальным (поскольку оно является топологически тривиальным расслоением на многообразии Штейна $\left.{ }^{15}\right)$, поэтому форма $\omega$ из $(0.6)$, построенная по голоморфной тривиализации этого расслоения, определяет систему (0.1) с заданными особыми точками и заданной монодромией. Затем Рёрль продолжает расслоение $F^{\prime}$ на всю сферу Римана $\overline{\mathbb{C}}$. Продолженное расслоение всегда имеет мероморфное сечение, голоморфно обратимое вне точек из $D$. Построенная по этому сечению система (0.1) имеет заданную монодромию, а точки $a_{1}, \ldots, a_{n}$ являются для нее регулярными особыми точками.

\footnotetext{
${ }^{11}$ Крылов Б. Л. Решение в конечном виде проблемы Римана для системы Гаусса // Труды Казан. авиац. ин-та, 1956, 31, 203-445.

${ }^{12}$ Еругин Н. П. Проблема Римана. Минск: Наука и техника, 1982.

${ }^{13}$ Röhrl H. Das Riemann-Hilbertsche Problem der Theorie der linearen Differentialgleichungen // Math. Ann., 1957, 133(1), 1-25.

${ }^{14}$ Birkhoff G. D. A theorem on matrices of analytic functions // Math. Ann., 1913, 74, 122-133.

${ }^{15}$ Grauert $H$. Analytische Faserungen uber holomorph-vollstandingen Raumen // Math. Ann., 1958, 135(3), 263-273.
} 
Таким образом, Рёрль передоказал результаты Племеля, а также доказал разрешимость проблемы Римана-Гильберта на некомпактной римановой поверхности. Кроме того, Рёрль доказал разрешимость в классе систем с регулярными особыми точками проблемы, аналогичной проблеме Римана-Гильберта, для произвольной римановой поверхности (при этом, правда, у построенной системы появляются, вообще говоря, дополнительные “ложные" особенности, не дающие вклада в монодромию).

В 1979 году появилась работа В. Деккерса ${ }^{16}$, из результатов которой вытекает разрешимость проблемы Римана-Гильберта для любого набора точек $a_{1}, \ldots, a_{n}$ и любого представления (0.4) размерности $p=2$. Различные аспекты проблемы РиманаГильберта и связанные с ней задачи рассматривались также в работах ${ }^{17}$.

3. Результаты по проблеме Римана-Гильберта представлены в главе 1. Основным результатом здесь является следующий:

ТЕОрема 1. Для любого $n>3$, любого набора точек $a_{1}, \ldots, a_{n} u$ любого $p \geqslant 3$ найдется такое представление (0.4), для которого не существует реализующей его фуксовой системы.

Теорема 1 означает, что проблема Римана-Гильберта имеет в общем случае отрицательное решение.

Отрицательный ответ на проблему, поставленную Гильбертом, порождает следующий вопрос: какие представления монодромии все-таки могут быть реализованы фуксовыми системами? Здесь главным является следующий результат, полученный в [3].

Теорема 2. Любое неприводимое представление (0.4) может быть реализовано как представление монодромии некоторой фуксовой системы ${ }^{18}$.

${ }^{16}$ Dekkers $W$. The matrix of a connection having regular singularities on a vector bundle of rank 2 on $P^{1}(C) / /$ Lecture Notes in Math., 1979, 712, 33-43.

${ }^{17}$ Голубева В. А. Некоторые вопросы аналитической теории фейнмановских интегралов // УМН, 1976, 31(2), 135-202; Сато М., Дзимбо М., Мива Т. Голономные квантовые поля. М.: Мир, 1983; Jimbo M., Miwa T. Monodromy preserving deformations of linear ordinary differential equations with rational coefficients. II // Physica D, 1981, 2, 407-448.

18 Этот результат был получен независимо также В. Костовым в Kostov V.P. Fuchsian systems on $C P^{1}$ and the Riemann-Hilbert problem // C. R. Acad. Sci. Paris Sér. I Math., 1992, 315, 143-148. 
Метод исследования проблемы Римана-Гильберта, обобщающий подходы Рёрля и Делиня ${ }^{19}$, состоит в следующем: вначале мы строим расслоение (точнее, серию расслоений) на сфере Римана с логарифмической связностью, имеющей заданную монодромию и заданные особые точки. Тем самым, исходная задача сводится к вопросу о тривиальности этого расслоения (точнее, какого-либо из построенных расслоений), ибо в тривиальном расслоении логарифмическая связность задает фуксову систему дифференциальных уравнений.

Затем, мы исследуем вопрос о тривиальности построенного расслоения (серии расслоений) и получаем условия разрешимости проблемы Римана-Гильберта.

Как известно, задача построения фуксова линейного дифференциального уравнения $p$-го порядка с данной монодромией и данными особыми точками имеет в общем случае при $p>1$ отрицательное решение, так как такое уравнение содержит меньше параметров, чем множество классов эквивалентности представлений (0.4) (такой подсчет параметров восходит к Пуанкаре ${ }^{20}$ ). Поэтому при построении фуксова уравнения возникают дополнительные особые точки коэффициентов, которые не дают вклада в монодромию и называются "ложными" особыми точками. В разделе 1.3 главы 1 найдено точное значение для минимально возможного числа $m_{0}$ таких точек.

По любому фуксову в окрестности точки $a_{i}$ уравнению с помощью специальной замены можно построить фуксову систему, однако это построение носит существенно локальный характер ${ }^{21}$. В работе [2] доказано следующее утверждение.

Теорема 3. По любому фуксову уравнению на сфере Римана можно построить фуксову систему (0.3) с теми же особыми точками и той же монодромией.

В разделе 1.2 главы 1 сформулированы также различные достаточные условия реализуемости представления (0.4) фуксовой системой.

${ }^{19}$ Deligne $P$. Equations différentielles a points singuliers réguliers // Lecture Notes in Math., 1970, 163, 1-133.

${ }^{20}$ Пуанкаре A. О группах линейных уравнений. М.: Наука, 1974. (Избр. тр. T. 3.)

${ }^{21}$ Хартман Ф. Обыкновенные дифференциальные уравнения. М.: Мир, 1970. 
В разделе 1.1 этой главы представлено краткое описание устройства пространства решений дифференциального уравнения с регулярными особыми точками, принадлежащее А.Х. М. Левелю ${ }^{22}$.

4. Методы, развитые для исследования проблемы РиманаГильберта, находят применение и в других задачах аналитической теории дифференциальных уравнений. К их числу относится задача о биркгофовой стандартной форме.

Рассмотрим в окрестности бесконечно удаленной точки систему линейных дифференциальных уравнений

$$
z \frac{d y}{d z}=C(z) y
$$

с матрицей коэффициентов $C(z)$ размера $(p, p)$ и вида

$$
C(z)=z^{r} \sum_{n=0}^{\infty} C_{n} z^{-n}, \quad C_{0} \neq 0, \quad r \geqslant 0,
$$

где ряд сходится в некоторой окрестности $O_{\infty}=\{z \in \mathbb{C}:|z|>R\}$ точки $\infty$.

Напомним, что число $r$ называется рангом Пуанкаре системы $(0.7)$ в особой точке $\infty$. Если $r>0$, то эта особая точка является, вообще говоря, иррегулярной особой точкой.

Под действием преобразования

$$
x=\Gamma(z) y
$$

система (0.7) переходит в систему

где

$$
z \frac{d x}{d z}=\widetilde{C}(z) x
$$

$$
\widetilde{C}(z)=z \frac{d \Gamma}{d z} \Gamma^{-1}+\Gamma C(z) \Gamma^{-1} .
$$

Если $\Gamma(z)$ голоморфно обратимо в $O_{\infty}$, то $\Gamma(z)$ называется аналитическим преобразованием. Если же $\Gamma(z)$ аналитично в $O_{\infty}$, а функции $\Gamma, \Gamma^{-1}$ лишь мероморфны в точке $\infty$, то $\Gamma(z)$ называется мероморфным преобразованием. Ясно, что аналитическое преобразование, в отличие от мероморфного, не меняет ранга Пуанкаре системы.

\footnotetext{
${ }^{22}$ Levelt A.H. M. Hypergeometric functions // Nederl. Akad. Wet., Proc.,
} Ser. A, 1961, 64, 361-401. (См. также [1], [5].) 
В 1913 году Биркгоф 23 доказал, что любая система (0.7) аналитическим преобразованием может быть приведена к такой системе $(0.10)$, в которой матрица коэффициентов $\widetilde{C}(z)$ имеет вид

$$
\widetilde{C}(z)=\widetilde{C}_{r} z^{r}+\cdots+\widetilde{C}_{0}
$$

т.е. $\widetilde{C}(z)$ является многочленом степени $r$ от переменной $z$.

С тех пор система вида $(0.10),(0.12)$ называется биркгофовой стандартной формой исходной системы (0.7).

Однако доказательство Биркгофа оказалось ошибочным, и в начале 1950-х годов Гантмахер привел контрпример к утверждению Биркгофа ${ }^{24}$. Как оказалось, доказательство Биркгофа проходит лишь для случая, когда матрица монодромии системы (0.7) в точке $\infty$ может быть приведена к диагональному виду.

Однако позднее было установлено, что препятствием к аналитической редукции системы к биркгофовой стандартной форме является ее приводимость.

Система (0.7) называется приводимой, если с помощью аналитического преобразования (0.9) она может быть приведена к виду $(0.10)$ с матрицей коэффициентов $\widetilde{C}$, имеющей блочный верхнетреугольный вид

$$
\widetilde{C}(z)=\left(\begin{array}{cc}
C^{\prime} & * \\
0 & C^{\prime \prime}
\end{array}\right)
$$

В противном случае система называется неприводимой.

Основным результатом главы 2 является следующая теорема:

Теорема 4. Любая неприводимая система (0.7) может быть преобразована $к$ биркгофовой стандартной форме с помощью аналитического преобразования (0.9).

(Ранее, в случае системы двух уравнений аналогичный результат был получен Юркатом, Лутцем и Пейеримхофом ${ }^{25}$, а в случае трех уравнений - Бальзером ${ }^{26}$.)

${ }^{23}$ Birkhoff G. D. Collected mathematical papers // J. Amer. Math. Soc., 1950, 1, 259-306.

${ }^{24}$ Гантмахер Ф. Р. Теория матриц. М.: Наука, 1988.

${ }^{25}$ Jurkat W. B., Lutz D. A., Peyerimhoff A. Birkhoff invariants and effective calculations for meromorphic differential equations. I // J. Math. Anal. Appl., 1976, 53, 438-470; II // Houston J. Math., 1976, 2, 207-238.

${ }^{26}$ Balser $W$. Analytic transformation to Birkhoff standard form in dimension three // Funkcial. Ekvac., 1990, 33(1), 59-67. 
В представленном цикле работ рассматривается также вопрос о возможности преобразования системы линейных дифференциальных уравнений к биркгофовой стандартной форме с помощью мероморфного преобразования, не повышающего ранга Пуанкаре системы в особой точке.

Эта задача является естественным обобщением предыдущей на более широкий класс преобразований и представляет значительный интерес в связи с тем, что мероморфные преобразования не меняют ни матриц Стокса, ни монодромии системы. Вопрос о возможности мероморфного приведения системы к биркгофовой стандартной форме возникает при исследовании обратной задачи в дифференциальной теории Галуа, при вычислении матриц Стокса, при доказательстве свойства Пенлеве для изомонодромных деформаций линейных систем с иррегулярными особыми точками и т.п.

В 1976 году Юркат, Лутц и Пейеримхоф в уже цитированной работе доказали что задача приведения системы уравнений к биркгофовой стандартной форме мероморфным преобразованием в случае системы двух уравнений всегда имеет положительное решение. В 1989 году Бальзер ${ }^{27}$ доказал аналогичный результат для системы трех уравнений.

В работе [11] мы доказываем следующее утверждение.

ТЕОРЕма 5. Любая система (0.7) четьрех и пяти уравнений, имеющая не более двух неприводимых блоков, может быть приведена к биркгофовой стандартной форме с помощъю не повышающего ранга Пуанкаре мероморфного преобразования (0.9).

5. В главе 3 решается задача об описании изомонодромных деформаций фуксовых систем дифференциальных уравнений.

Наиболее известным видом изомонодромной деформации фуксовой системы

$$
\frac{d y}{d z}=\left(\sum_{i=1}^{n} \frac{B_{i}^{0}}{z-a_{i}^{0}}\right) y
$$

на сфере Римана является деформация, задаваемая уравнением Шлезингера. Она определяется пфаффовой системой

$$
d y=\omega_{s} y, \quad \omega_{s}=\sum_{i=1}^{n} \frac{B_{i}(a)}{z-a_{i}} d\left(z-a_{i}\right)
$$

${ }^{27}$ Balser $W$. Meromorphic transformation to Birkhoff standard form in dimension three // J. Fac. Sci. Univ. Tokyo Sect. IA Math., 1989, 36, 233-246. 
и начальными данными $\left.B_{i}(a)\right|_{a^{0}}=B_{i}^{0}$. Условие изомонодромности эквивалентно условию полной интегрируемости $d \omega_{s}=\omega_{s} \wedge \omega_{s}$ последней системы. Указанное условие имеет вид

$$
d B_{i}(a)=-\sum_{j=1, j \neq i}^{n} \frac{\left[B_{i}(a), B_{j}(a)\right]}{a_{i}-a_{j}} d\left(a_{i}-a_{j}\right) .
$$

Оно и называется уравнением Шлезингера изомонодромных деформаций.

Уравнение Шлезингера исследовалось самим Шлезингером ${ }^{28}$, Джимбо и Мивой ${ }^{29}$, Б. Мальгранжем ${ }^{30}$, Итсом и Новокшеновым $^{31}$, Сибуйей ${ }^{32}$ и другими математиками с различных точек зрения.

Оказывается, что если матрицы $B_{i}^{0}$ коэффициентов деформируемой системы не имеют резонансов, то любая изомонодромная деформация либо описывается уравнением Шлезингера, либо сводится к последнему голоморфной по параметру деформации заменой ${ }^{33} y^{\prime}=C(a) y$. Последние деформации полностью описываются вполне интегрируемыми пфаффовыми системами с формой коэффициентов

$$
\omega_{n}=\omega_{s}+\sum_{r=1}^{n} \gamma_{r}(a) d a_{r} .
$$

В настоящей работе мы приводим описание общего вида изомонодромных деформаций произвольной фуксовой системы при наличии резонансов. Напомним, что матрица коэффициентов $B_{i}^{0}$ называется резонансной, если для какой-либо пары ее собственных значений разность между этими собственными значениями

\footnotetext{
${ }^{28}$ Schlesinger L. Über Lösungen gewisser Differentialgleichungen als Funktionen der singularen Punkte // J. Reine Angew. Math., 1905, 129, 287-294.

29 Jimbo M., Miwa T. Monodromy preserving deformations of linear ordinary differential equations with rational coefficients. II // Physica D, 1981, 2, 407-448.

${ }^{30}$ Malgrange B. Sur les déformations isomonodromiques // Progr. Math., 1983, 37, 427-438.

${ }^{31}$ Its A., Novokshenov $V$. The isomonodromic deformation method in the theory of Painlevé equations // Lecture Notes in Math., 1986, 1191.

${ }^{32}$ Sibuya $S$. Linear differential equations in the complex domain: problems of analytic continuation. Providence, 1990.

${ }^{33}$ Iwasaki K. et al. From Gauss to Painlevè. A modern theory of special functions. Braunschweig: Vieweg, 1991.
} 
является натуральным числом. Наибольшее число $r_{i}$ из таких чисел называется максимальным $i$-резонансом системы.

Мы доказываем, что любая изомонодромная деформаиия фуксовой системы определяется вполне интегрируемой пфафбовой системой с формой коэффичиентов вида $\omega=\omega_{n}+\omega_{m}$, где $\omega_{m}$ некоторая мероморфная равная нулю при $z=\infty$ дифференциальная форма с полюсами порядка не выше, чем $r_{i}$ на $\left\{z-a_{i}=0\right\}$.

\section{1. Проблема Римана-Гильберта}

\section{§ 1.1. Локальное устройство фуксовой системы}

Рассмотрим пространство $X$ решений системы $(0.1)$ в окрестности регулярной особой точки $a_{i}$. Будем считать, что $a_{i}=0$ (для чего сделаем замену координат $\left.z \mapsto z-a_{i}\right)$. Выберем какую-либо фундаментальную матрицу $Y(z)$ пространства решений $X$ и обозначим через $G$ матрицу монодромии фундаментальной матрицы $Y(z)$ в точке 0.

Обозначим через $E$ матрицу $(1 / 2 \pi i) \ln G$. Выберем раз и навсегда собственные значения $\rho^{j}$ матрицы $E$ так, чтобы выполнялись неравенства

$$
0 \leqslant \operatorname{Re} \rho^{j}<1 .
$$

Определим матрицу $z^{E}$ следующим образом:

$$
z^{E}=\exp (E \ln z)=I+E \ln z+\cdots+E^{n} \ln ^{n} \frac{z}{n !}+\cdots .
$$

Тогда при аналитическом продолжении вдоль петли $\sigma$ матричная функция $z^{E}$ переходит в

$$
\exp (E(\ln z+2 \pi i))=z^{E} \exp (2 \pi i E)=z^{E} G,
$$

то есть матрица $z^{E}$ имеет ту же монодромию, что и исходная фундаментальная матрица $Y(z)$. Поэтому имеет место

ЛЕмма 1. Фундаментальная матрица $Y(z)$ имеет следующее разложение в проколотой окрестности $\dot{O}$ точки $z=0$ :

$$
Y(z)=M(z) z^{E}
$$

где $M(z)$ - однозначная матричная функиия в $\dot{O}$. 
Для дальнейшего нам понадобится следующая техническая лемма, касающаяся характера поведения матричной функции $z^{E}$ в окрестности нуля.

Лемма 2. Элементы $\left(\left(a_{i j}\right)\right)$ матрицы $z^{E}$ имеют вид

$$
a_{i j}=\sum_{l=1}^{p} z^{\rho^{l}} P_{i j}^{l}(\ln z),
$$

где $\rho^{l}-$ собственные значения матриць $E, a P_{i j}^{l}(\ln z)-$ многочлены от $\ln z$ степени не больше $p-1$.

СлЕДСТВИЕ 1. Точка $z=0$ является регулярной особой точкой для системъ (0.1) тогда и только тогда, когда матрица $M(z)$ в разложении (1.2) мероморфна в нуле.

На пространстве решений системы с регулярной особой точкой можно ввести понятие нормирования (целочисленной скорости роста решений) следующим образом. Из лемм 1 и 2 следует, что любое решение $y(z)$ может быть записано в виде так называемой конечной логарифмической суммы

$$
y(z)=\sum_{j, l \in \sigma} f_{j l}(z) z^{\rho_{j}}(\ln z)^{b_{l}},
$$

где $f_{j l}$ - ряды Лорана с конечной главной частью, числа $\rho_{j}$ удовлетворяют соотношению (1.1), $b_{l}$ - целые неотрицательные, и подобные члены (относительно пар $\left.\left(\rho_{j}, b_{l}\right)\right)$ приведены.

Нормированием решения $y(z)$ в нуле называется минимум нормирований рядов $f_{j l}$ по всем $j, l \in \sigma$, где, в свою очередъ, под нормированием функции $f_{j l}(z)$ в нуле понимается порядок ее нуля или порядок ее полюса (со знаком минус) в точке $z=0$.

Рассмотрим вновь пространство решений $X$ системы (0.1).

ПрЕДЛОЖЕНИЕ 1. Нормирование $\varphi$ задает отображение $\varphi$ : $X \rightarrow \mathbb{Z} \cup \infty$, обладающее следующими свойствами:

а) $\varphi\left(y_{1}+y_{2}\right) \geqslant \min \left(\varphi\left(y_{1}\right), \varphi\left(y_{2}\right)\right)$, причем если $\varphi\left(y_{1}\right) \neq \varphi\left(y_{2}\right)$, то имеет место равенство;

б) $\varphi(c y)=\varphi(y)$ для любого $c \in \mathbb{C} \backslash 0$;

в) $\varphi\left(\sigma^{*} y\right)=\varphi(y)$, где $\sigma^{*}$ - оператор монодромии системы в особой точке $z=0$. 
Из свойств а) и б) следует, что нормирование $\varphi$ принимает на $X$ конечное число значений $\infty>\psi^{1}>\cdots>\psi^{m}$ и задает фильтрацию

$$
0 \subset X^{1} \subset \cdots \subset X^{m}=X
$$

пространства $X$ линейными подпространствами

$$
X^{k}=\left\{y \in X \mid \varphi(y) \geqslant \psi^{k}\right\}, \quad k=1, \ldots, m .
$$

Согласно свойству в) оператор $\sigma^{*}$ сохраняет эту фильтрацию. Обозначим через $k_{l}$ размерность факторпространства $X^{l} / X^{l-1}$, а через ${ }^{l} \sigma^{*}$ - ограничение $\sigma^{*}$ на $X^{l}$.

Рассмотрим базис $e_{1}^{1}, \ldots, e_{k_{1}}^{1}$ пространства $X^{1}$, в котором матрица оператора ${ }^{1} \sigma^{*}$ имеет верхний треугольный вид, дополним его до базиса $e_{1}^{1}, \ldots, e_{k_{1}}^{1}, e_{1}^{2}, \ldots, e_{k_{2}}^{2}$ пространства $X^{2}$, в котором верхний треугольный вид имеет матрица оператора ${ }^{2} \sigma^{*}$ и т.д. (Для построения элементов $e_{1}^{l}, \ldots, e_{k_{l}}^{l}$ достаточно рассмотреть произвольный базис $\bar{e}_{1}^{l}, \ldots, \bar{e}_{k_{l}}^{l}$ факторпространства $X^{l} / X^{l-1}$, в котором матрица индуцированного оператором ${ }^{l} \sigma^{*}$ оператора ${ }^{l, l-1} \sigma^{*}: X^{l} / X^{l-1} \rightarrow X^{l} / X^{l-1}$, имеет верхний треугольный вид, а затем выбрать произвольных представителей этого базиса в $X^{l}$.)

Построенный базис $(e)=\left(e_{1}, \ldots, e_{p}\right)$ пространства $X$ обладает следующими свойствами:

1) нормирование $\varphi$ принимает на элементах базиса $(e)$ все свои значения $\psi^{1}, \ldots, \psi^{m}$ (с учетом кратностей $k_{1}, \ldots, k_{m}$ );

2) $\varphi\left(e_{l+1}\right) \leqslant \varphi\left(e_{l}\right), \quad l=1, \ldots, p-1$;

$3)$ матрица $G$ оператора $\sigma^{*}$ имеет в этом базисе верхний треугольный вид.

ОПрЕДЕЛЕНИЕ 1. Базис $(e)$ пространства $X$ решений системы (0.1) с регулярной особой точкой 0 , удовлетворяющий свойствам 1)-3), называется левелевским.

ПримеР 1. Пусть оператор $\sigma^{*}$ приводится к жордановой клетке. Тогда соответствующий жорданов базис $(e)$ будет левелевским базисом пространства $X$.

Любой другой левелевский базис пространства $X$ может быть получен из $(e)$ верхнетреугольным преобразованием. 
ДокАзАТЕльство. Обозначим через $Y^{l}$ подпространство размерности $l$ пространства $X$, натянутое на векторы $e_{1}, \ldots, e_{l}$ жорданова базиса $(e)$. Фильтрация

$$
0 \subset Y^{1} \subset \cdots \subset Y^{p}=X
$$

является единственной фильтрацией длины $p$ пространства $X$, инвариантной относительно действия $\sigma^{*}$, поскольку элементы $e_{1}, \ldots, e_{p}$ образуют цепочку присоединенных векторов, т.е.

$$
\sigma^{*}\left(e_{l}\right)=\lambda e_{l}+e_{l-1}, \quad l=2, \ldots, p, \quad \lambda \in \mathbb{C} \backslash 0 .
$$

Другими словами, любое подпространство $X^{l}$ пространства $X$ размерности $d_{l}$, инвариантное относительно действия $\sigma^{*}$, совпадает с $Y^{l}$. Отсюда немедленно следует, что жорданов базис $(e)$ ассоциирован с фильтрацией (1.4), т.е. удовлетворяет свойствам 1), 2). Так как (e) удовлетворяет и свойству 3$)$, то $(e)$ - левелевский базис пространства $X$.

Вторая часть утверждения следует из того, что в условиях примера любой базис, в котором матрица оператора монодромии имеет верхнетреугольный вид, может быть получен из жорданового некоторым верхнетреугольным преобразованием.

Рассмотрим пространство решений $X$ системы (0.1) с регулярной особой точкой $z=0$ и произвольный левелевский базис $(e)$ пространства $X$. Обозначим через $G$ матрицу оператора $\sigma^{*}$ в базисе $(e)$, а через $A=\operatorname{diag}\left(\varphi^{1}, \ldots, \varphi^{p}\right)$ - диагональную целочисленную матрицу нормирований этого базиса, т.е. $\varphi^{l}=\varphi\left(e_{l}\right)$. Рассмотрим матрицу $E$ из (1.1). Имеет место следующее утверждение из [1].

Лемма 3. Матрицы $z^{A} G z^{-A}$ и $z^{A} E z^{-A}$ голоморфны в точке $z=0$. Нормирование матричной функции $z^{A} z^{E} z^{-A}$ в точке 0 равно нулю.

Теорема 6. Для фундаментальной матрицы $Y_{e}(z)$, построенной по левелевскому базису (e) пространства решений $X$ системъ (0.1) в $\dot{O}$ с регулярной особой точкой 0 , имеет место следующее разложение:

$$
Y_{e}(z)=U(z) z^{A} z^{E},
$$

где матрица $U(z)$ однозначна и голоморфна в окрестности точки 0. 
Следующая теорема, принадлежащая голландскому математику А.Х. М. Левелю ${ }^{34}$, выделяет фуксовы системы среди всех систем с регулярной особой точкой.

ТЕОРема 7. Система (0.1) с регулярной особой точкой $z=0$ фуксова в этой точке тогда и только тогда, когда в разложении (1.5)

$$
Y_{e}(z)=U(z) z^{A} z^{E}
$$

для фундаментальной матрицъ $Y_{e}(z)$, построенной по левелевскому базису (е) пространства решений $X$ системь (0.1) в $\dot{O}$, матрица $U(z)$ голоморфно обратима в окрестности точки 0.

Числа $\varphi^{j}+\rho^{j}=\beta^{j}$ называются показателями системы в регулярной особой точке 0. Согласно последней теореме они задают асимптотики решений фуксовой системы в этой особой точке.

В качестве немедленного следствия получаем следующее утверждение.

СЛЕДСТвиЕ 2. Показатели фуксовой системъ

$$
\frac{d y}{d z}=\frac{B_{0}(z)}{z} y
$$

в нуле совпадают с собственными значениями матриць $B_{0}(0)$ коэффиииентов этой системы.

\section{§ 1.2. Метод решения. Достаточные условия разрешимости}

Первый этап в решении проблемы Римана-Гильберта состоит в построении на проколотой сфере Римана $B=\overline{\mathbb{C}} \backslash\left\{a_{1}, \ldots, a_{n}\right\}$ расслоения $F$ с голоморфной связностью $\nabla$, имеющей заданную монодромию (0.4). Напомним эту хорошо известную конструкцию ${ }^{35}$.

Рассмотрим конечное покрытие пространства $B$ связными односвязными окрестностями $\left\{U_{i}\right\}$ со связными односвязными пересечениями. Выберем в каждой окрестности по точке $z_{0}^{i}$ и

\footnotetext{
${ }^{34}$ Levelt A.H. M. Hypergeometric functions // Nederl. Akad. Wet., Proc., Ser. A, 1961, 64, 361-401.

${ }^{35}$ Atiyah M. F. Complex analytic connections in fibre bundles // Trans. Amer. Math. Soc., 1957, 85(1), 181-207.
} 
соединим точку $z_{0}$ с этими точками путями $\eta_{i}$. Внутри каждого множества $U_{i} \cup U_{j}$ с непустым пересечением $U_{i} \cap U_{j}$ соединим точки $z_{0}^{i}$ и $z_{0}^{j}$ путем $\delta_{i j}$. Обозначим через $g_{i j}$ матрицу

$$
g_{i j}=\chi\left(\left[\eta_{i} \circ \delta_{i j} \circ \eta_{j}^{-1}\right]\right),
$$

где через $\xi \circ \zeta$ обозначается путь, являющийся результатом последовательного обхода путей $\xi$ и $\zeta$ (предполагается, что конец пути $\xi$ совпадает с началом пути $\zeta$ ), а через $\xi^{-1}-$ путь $\xi$, проходимый в обратном направлении. Гомотопический класс пути $\xi$ обозначается через $[\xi]$ и гомотопический класс постоянной петли $z_{0}$ через $e$.

Ясно, что $g_{i j}=\left(g_{j i}\right)^{-1}$ и $g_{i j} g_{j k} g_{k i}=I$ в случае непустого пересечения $U_{i} \cap U_{j} \cap U_{k}$. Действительно, в этом случае

$$
\begin{aligned}
& \chi\left(\left[\eta_{i} \circ \delta_{i j} \circ \eta_{j}^{-1}\right]\right) \chi\left(\left[\eta_{j} \circ \delta_{j k} \circ \eta_{k}^{-1}\right]\right) \chi\left(\left[\eta_{k} \circ \delta_{k i} \circ \eta_{i}^{-1}\right]\right) \\
& \quad=\chi\left(\left[\eta_{i} \circ \delta_{i j} \circ \eta_{j}^{-1} \circ \eta_{j} \circ \delta_{j k} \circ \eta_{k}^{-1} \circ \eta_{k} \circ \delta_{k i} \circ \eta_{i}^{-1}\right]\right) \\
& \quad=\chi\left(\left[\eta_{i} \circ \delta_{i j} \circ \delta_{j k} \circ \delta_{k i} \circ \eta_{i}^{-1}\right]\right)=\chi(e)=I,
\end{aligned}
$$

так как петля $\delta_{i j} \circ \delta_{j k} \circ \delta_{k i}$ гомотопна постоянной петле (так как $U_{i} \cap U_{j} \cap U_{k}$ односвязно).

Рассмотрим векторное расслоение $F$ на $B$, построенное по покрытию $\left\{U_{i}\right\}$ и постоянному коциклу $\left\{g_{i j}\right\}$. Введем в расслоении $F$ голоморфную связность $\nabla$, задав ее нулевыми формами $\omega_{i}=0$ в данном координатном описании расслоения $F$. Имеет место следующее утверждение:

ПрЕДЛОЖЕНИЕ 2. Построенная связность $\nabla$ имеет заданную монодромию (0.4).

ДоказАтельство. Рассмотрим петлю $\gamma$, лежащую в $B$ и конечное покрытие этой петли окрестностями $U_{1}, \ldots, U_{m}$. Можно считать, что $z_{0} \in U_{1}$ и $\eta_{1}=z_{0}$. Монодромия связности вдоль пути $\gamma$ равна $G_{\gamma}=\left(g_{1 m} g_{m m-1} \cdots g_{21}\right)^{-1}$. Но в нашем случае

$$
\begin{gathered}
\left(g_{1 m} g_{m m-1} \cdots g_{21}\right)^{-1}=g_{21}^{-1} \cdots g_{m m-1}^{-1} g_{1 m}^{-1}=g_{12} \cdots g_{m-1 m} g_{m 1} \\
=\chi\left(\left[\eta_{1} \circ \delta_{12} \circ \eta_{2}^{-1}\right]\right) \cdots \chi\left(\left[\eta_{m-1} \circ \delta_{m-1 m} \circ \eta_{m}^{-1}\right]\right) \\
\quad \times \chi\left(\left[\eta_{m} \circ \delta_{m 1} \circ \eta_{1}^{-1}\right]\right)
\end{gathered}
$$




$$
\begin{aligned}
& =\chi\left(\left[\eta_{1} \circ \delta_{12} \circ \eta_{2}^{-1} \circ \cdots \circ \eta_{m-1} \circ \delta_{m-1 m} \circ \eta_{m}^{-1} \circ \eta_{m} \circ \delta_{m 1} \circ \eta_{1}^{-1}\right]\right) \\
& \left.=\chi\left(\left[\eta_{1} \circ \delta_{12} \circ \cdots \circ \delta_{m-1 m} \circ \delta_{m 1} \circ \eta_{1}^{-1}\right]\right]\right)=\chi([\gamma]),
\end{aligned}
$$

так как петля $\delta_{12} \circ \cdots \circ \delta_{m-1 m} \circ \delta_{m 1}$ гомотопна петле $\gamma$.

Легко доказывается следующее утверждение.

ЛЕмма 4. Любое голоморфное векторное расслоение на проколотой сфере Римана $B$ с голоморфной связностью, имеющей монодромию (0.4), эквивалентно расслоению $(F, \nabla)$.

Следующий этап состоит в продолжении построенного расслоения на всю сферу Римана до расслоения с логарифмической связностью $\nabla^{\prime}$, совпадающей с исходной связностью вне особых точек $a_{1}, \ldots, a_{n}$.

Рассмотрим окрестность $U_{\alpha}$ нашего покрытия, граница которой содержит точку $a_{i}$. Рассмотрим базис горизонтальных сечений $\left(e_{1}^{\alpha}, \ldots, e_{p}^{\alpha}\right)$ связности $\nabla$ над $U_{\alpha}$. Поскольку монодромия связности $\nabla$ по построению совпадает с $(0.4)$, то при аналитическом продолжении вдоль малой петли $\delta_{i}$, обходящей точку $a_{i}$ против часовой стрелки этот базис перейдет в базис $\left(e_{1}^{\alpha}, \ldots, e_{p}^{\alpha}\right) G_{i}$, где $G_{i}$ - матрица, сопряженная к матрице монодромии в точке $a_{i}$. Рассмотрим функцию $\left(z-a_{i}\right)^{-E_{i}}$ в окрестности $U_{\alpha}$, точнее, некоторую ветвь этой функции.

Сечения $\left(\xi_{1}^{0}, \ldots, \xi_{p}^{0}\right)=\left(e_{1}^{\alpha}, \ldots, e_{p}^{\alpha}\right)\left(z-a_{i}\right)^{-E_{i}}$ (где собственные значения матрицы $E_{i}=(1 / 2 \pi i) \ln G_{i}$ нормализованы согласно неравенствам (1.1)) образуют базис голоморфных сечений расслоения $F$ над $O_{i} \backslash\left\{a_{i}\right\}$ (и, значит, расслоение $F$ голоморфнотривиально над $O_{i} \backslash\left\{a_{i}\right\}$ ). (Действительно, при продолжении вдоль $\delta_{i}$ этот базис переходит в себя.) Сечения $\left(\xi_{1}^{0}, \ldots, \xi_{p}^{0}\right)$ уже не являются горизонтальными, и форма связности $\nabla$ в этом базисе имеет вид

$$
\omega^{\xi}=E_{i} \frac{d z}{z-a_{i}} .
$$

Продолжим расслоение $F$ в точку $a_{i}$ следующим образом. Рассмотрим цилиндр $O_{i} \times \mathbb{C}^{p}$ (тривиальное векторное расслоение над $\left.O_{i}\right)$ с базисом сечений $\left(s_{1}, \ldots, s_{p}\right)$, где $s_{i}=\left(z, e_{i}\right)$, а $e_{i}-i$-й элемент стандартного базиса пространства $\mathbb{C}^{p}$.

Приклеим цилиндр $O_{i} \times \mathbb{C}^{p}$ к пространству расслоения $F_{E}$, отождествив при всех $i$ сечения $\xi_{i}^{0}$ с $s_{i}$ над $O_{i} \backslash\left\{a_{i}\right\}$ и продолжив 
склейку на $\left(O_{i} \backslash\left\{a_{i}\right\}\right) \times \mathbb{C}^{p}$ по линейности. Получим расслоение над $B \cup O_{i}$. Какой вид имеет координатное описание этого расслоения в окрестности точки $a_{i}$ ?

Обозначим окрестность $O_{i}$ через $U_{0}$. Если в качестве исходного базиса $\left(e^{\alpha}\right)=\left(e_{1}^{\alpha}, \ldots, e_{p}^{\alpha}\right)$ выбран базис, соответствующий исходной тривиализации расслоения $F$ (т.е. $\left(e^{\alpha}\right) g_{\alpha \beta}=\left(e^{\beta}\right)$ для непустых $\left.U_{\alpha} \cap U_{\beta}\right)$, то по построению $g_{0 \alpha}(z)=\left(z-a_{i}\right)^{E_{i}}$. Для любой другой окрестности $U_{\beta}$, содержащей точку $a_{i}$ в своем замыкании, $g_{0 \beta}(z)$ является результатом аналитического продолжения функции $g_{0 \alpha}(z)$ в $U_{\beta}$ вдоль пути $\delta_{i}$. (После такого продолжения, вернувшись в $U_{\alpha}$, получим вместо выбранной первоначально ветви $\left(z-a_{i}\right)^{E_{i}}$ ветвь $\left(z-a_{i}\right)^{E_{i}} G_{i}$, что и обеспечивает корректность продолжения расслоения.

Любой другой базис $\left(\widetilde{e}^{\alpha}\right)$ горизонтальных сечений связан с базисом $\left(e^{\alpha}\right)$ соотношением $\left(\widetilde{e}^{\alpha}\right)=\left(e^{\alpha}\right) S_{i}$ и при продолжении вдоль $\delta_{i}$ переходит в $\left(\widetilde{e}^{\alpha}\right) \widetilde{G}_{i}$, где $\widetilde{G}_{i}=S_{i}^{-1} G_{i} S_{i}$. Выберем такой базис $\left(\widetilde{e}^{\alpha}\right)$, для которого матрица $\widetilde{G}_{i}$ является верхнетреугольной. Обозначим через $\Lambda_{i}$ диагональную матрицу с целочисленными элементами $\lambda_{i}^{j}, j=1, \ldots, p$, образующими невозрастающую последовательность: $\lambda_{i}^{j} \geqslant \lambda_{i}^{j+1}, j=1, \ldots, p-1$. Будем называть такие матрицы допустимыми.

Рассмотрим базис локальных сечений $\left(\xi^{\Lambda_{i}}\right)=\left(\widetilde{e}^{\alpha}\right)\left(z-a_{i}\right)^{-\widetilde{E}_{i}} \times$ $\left(z-a_{i}\right)^{-\Lambda_{i}}$ расслоения $F$ над $O_{i} \backslash\left\{a_{i}\right\}$ и продолжим расслоение $F$ в точку $a_{i}$ аналогично тому, как это было сделано выше, заменив сечения базиса $\left(\xi^{0}\right)$ на $\left(\xi^{\Lambda_{i}}\right)$. Форма $\omega^{\Lambda_{i}}$ связности $\nabla$ в этом базисе имеет вид

$$
\omega^{\Lambda_{i}}=\left(\Lambda_{i}+\left(z-a_{i}\right)^{\Lambda_{i}} \widetilde{E}_{i}\left(z-a_{i}\right)^{-\Lambda_{i}}\right) \frac{d z}{z-a_{i}} .
$$

Так как матрица $\left(z-a_{i}\right)^{\Lambda_{i}} \widetilde{E}_{i}\left(z-a_{i}\right)^{-\Lambda_{i}}$ голоморфна в точке $a_{i}$ (см. лемму 3 ), то форма $\omega^{\Lambda_{i}}$ имеет логарифмическую особенность в этой точке.

Функция перехода $\widetilde{g}_{0 \alpha}$ построенного расслоения (в исходном координатном описании) записывается в виде

$$
\widetilde{g}_{0 \alpha}=\left(z-a_{i}\right)^{\Lambda_{i}}\left(z-a_{i}\right)^{\widetilde{E}_{i}} S_{i}^{-1}=\left(z-a_{i}\right)^{\Lambda_{i}} S_{i}^{-1}\left(z-a_{i}\right)^{E_{i}} .
$$


Рассмотрим набор $\Lambda=\left(\Lambda_{1}, \ldots, \Lambda_{n}\right)$, состоящий из допустимых матриц $\Lambda_{1}, \ldots, \Lambda_{n}$ (будем называть такой набор допустимымм). Продолжим расслоение $F$ в каждую точку $a_{i}$ с помощью соответствующей матрицы $\Lambda_{i}$, получим голоморфное векторное расслоение $F^{\Lambda}$ на всей сфере Римана с логарифмической связностью $\nabla^{\Lambda}$. Обозначим множество всех таких расслоений через $\mathcal{F}$.

Расслоение $F^{0}$ со связностью $\nabla^{0}$ (т.е., продолжение, построенное по набору $\Lambda_{1}=\cdots=\Lambda_{n}=0$ ) называется каноническим продолжением исходного расслоения $(F, \nabla)$.

Продолжение $\left(F^{\Lambda}, \nabla^{\Lambda}\right)$ расслоения $(F, \nabla)$ зависит также от выбора матриц $S_{1}, \ldots, S_{n}$ (от способа приведения матриц монодромии к верхнетреугольной форме), от исходного координатного описания расслоения $F$ и от выбора ветвей функций $\left(z-a_{i}\right)^{E_{i}}$. Последние две зависимости несущественны, так как они сводятся к соответствующему изменению матриц $S_{1}, \ldots, S_{n}$. Что касается зависимости расслоения от $S_{1}, \ldots, S_{n}$, то она, действительно, очень важна. Два расслоения, построенные по одному и тому же допустимому набору $\Lambda$, но по разным матрицам $S_{i}$, вообще говоря, могут быть не эквивалентны.

Однако, в дальнейшем для краткости изложения мы будем опускать эту зависимость (постоянно “держа" ее в уме).

Все сказанное выше не относится к каноническому продолжению $\left(F^{0}, \nabla^{0}\right)$, которое зависит лишь от исходного представления (0.4).

Следующее утверждение является немедленным следствием теоремы 7 и вышеприведенной конструкции.

ПРЕДЛОЖЕНИЕ 3. Множество $\mathcal{F}$ содержит все голоморфные векторные расслоения с логарифмическими связностями на сфере Римана с данными особыми точками и данной монодромией.

Поскольку фуксова система на сфере Римана с данными особыми точками и монодромией может быть рассмотрена как связность в некотором тривиальном расслоении, в качестве следствия получаем следующее утверждение.

Теорема 8. Представление (0.4) может быть реализовано в качестве представления монодромии некоторой фуксовой системы на сфере Римана с заданными особыми точками $a_{1}, \ldots, a_{n}$ тогда и только тогда, когда множество $\mathcal{F}$ расслоений, построенных по представлению (0.4), содержит хотя бы одно голоморфно тривиальное расслоение. 
Таким образом, мы свели проблему Римана-Гильберта к исследованию вопроса о голоморфной тривиальности некоторого расслоения (множества расслоений) на сфере Римана.

Как известно ${ }^{36}$, любое голоморфное расслоение $E$ на сфере Римана раскладывается в прямую сумму одномерных

$$
E \cong \mathcal{O}\left(k_{1}\right) \oplus \cdots \oplus \mathcal{O}\left(k_{p}\right)
$$

где числа $k_{i}$ целые, $k_{1} \geqslant \cdots \geqslant k_{p}$, и расслоение $\mathcal{O}(k)$ задается следующим координатным описанием $\mathcal{O}(k)=\left(\mathbb{C}, \overline{\mathbb{C}} \backslash 0, g_{0 \infty}=z^{k}\right)$.

Из этой теоремы (называемой теоремой Биркгофа-Гротендика) немедленно следует, что каждое расслоение на сфере Римана мероморфно тривиально. Точнее, имеет место следующее утверждение.

ПрЕДЛОЖЕНИЕ 4. У любого голоморбного векторного расслоения Е на сфере Римана существует базис мероморфных сечений, голоморфный вне одной произвольной наперед заданной точки.

ДокАзАтельство. Рассмотрим координатное описание (1.10) расслоения $E$ и произвольную точку $b \in \mathbb{C}$. Функции $w_{i}=$ $((z-b) / z)^{k_{i}}, \quad v_{i}=(z-b)^{k_{i}}$ голоморфны в $\quad \overline{\mathbb{C}} \backslash 0 \quad$ и $\quad \mathbb{C}$ соответственно (за исключением точки $b$ ), и столбцы матриц $W=\operatorname{diag}\left(w_{1}, \ldots, w_{p}\right), V=\operatorname{diag}\left(v_{1}, \ldots, v_{p}\right)$ задают координатное описание требуемого базиса (так как $V=g_{0 \infty} W$ ).

В качестве следствия получаем следующее утверждение, принадлежащее Племелю.

ТЕОрема 9. (Племель) Любое представление (0.4) может быть реализовано как представление монодромии некоторой системы с регулярными особыми точками, фуксовой во всех точках кроме, быть может, одной, с любыми наперед заданными допустимыми нормированиями в фуксовых точках.

\footnotetext{
${ }^{36}$ Оконек К., Шнейдер М., Шпиндлер X. Векторные расслоения на комплексном проективном пространстве. М.: Мир, 1984.
} 
Доказательство. Рассмотрим произвольное расслоение $E$ из построенного множества $\mathcal{F}$ расслоений с данной монодромией и данными особыми точками. Положим $b=a_{i}$ и рассмотрим базис $(e)=\left(e_{1}, \ldots, e_{p}\right)$ сечений этого расслоения, построенный в предыдущем предложении.

В базисе из этих сечений логарифмическая связность $\nabla$ определяет систему линейных дифференциальных уравнений с данными особыми точками и монодромией, фуксовую во всех особых точках и имеющую регулярную особенность в точке $a_{i}$.

Действительно, так как этот базис лишь мероморфен в точке $a_{i}$, то $\left(\xi^{\Lambda_{i}}\right)=(e) U(z)$, где $\left(\xi^{\Lambda_{i}}\right)$ - голоморфный базис локальных сечений расслоения $E$ над окрестностью $O_{i}$ точки $a_{i}$, задающий продолжений расслоения $F$, построенного по монодромии $(0.4)$, в точку $a_{i}$, и матричная функция $U(z)$ мероморфна в $a_{i}$.

Поэтому согласно (1.9) форма $\omega_{i}$ связности имеет в $O_{i}$ следующий вид

$$
\omega_{i}=d U U^{-1}+U\left(\Lambda_{i}+\left(z-a_{i}\right)^{\Lambda_{i}} \widetilde{E}_{i}\left(z-a_{i}\right)^{-\Lambda_{i}}\right) U^{-1} \frac{d z}{z-a_{i}} .
$$

А фундаментальная матрица пространства решений этой системы (горизонтальных сечений связности) вблизи точки $a_{i}$ записывается следующим образом

$$
Y_{i}(z)=U(z)\left(z-a_{i}\right)^{\Lambda_{i}}\left(z-a_{i}\right)^{\widetilde{E}_{i}} .
$$

Поэтому точка $a_{i}-$ регулярная особая для построенной системы. В силу произвольности выбранного расслоения из $\mathcal{F}$ нормирования в точках, отличных от $a_{i}$, могут быть выбраны произвольными допустимыми.

Набор чисел $k_{1}, \ldots, k_{p}$ называется типом расщепления расслоения. Он полностью характеризует голоморфный тип расслоения, в то время как сумма этих чисел, называемая степенъю расслоения $E$ полностью характеризует его топологический тип. Таким образом, расслоение $E$ голоморфно тривиально тогда и только тогда, когда все числа $k_{1}, \ldots, k_{p}$ равны нулю.

В теории интегральных уравнений эти числа называются частными индексами, и как хорошо известно, в общем случае их вычислить невозможно. Однако, для некоторых специальных классов представлений (0.4) об этих числах можно получить некоторую информацию, достаточную для решения проблемы Римана-Гильберта. 
Теорема 10. Рассмотрим расслоение $E \in \mathcal{F}$ с логарифмической связностъю $\nabla$, построенное по неприводимому представлению (0.4) с особыми точками $a_{1}, \ldots, a_{n}$. Для типа расщепления этого расслоения имеют место следующие неравенства

$$
k_{i}-k_{i+1} \leqslant n-2, \quad i=1, \ldots, p-1 .
$$

ДокАЗАтельство. Рассмотрим систему дифференциальных уравнений с заданной монодромией и особыми точками, форма коэффициентов $\omega^{\prime}$ которой имеет простые полюса во всех особых точках кроме $a_{i}$, а в окрестности этой точки имеет вид

$$
\omega^{\prime}=-\frac{K}{z-a_{i}} d z+\left(z-a_{i}\right)^{-K} \omega\left(z-a_{i}\right)^{K},
$$

а фундаментальная матрица пространства решений системы

$$
d y=\omega^{\prime} y
$$

в окрестности точки $a_{i}$ представляется в виде

$$
Y_{i}(z)=\left(z-a_{i}\right)^{-K} V(z)\left(z-a_{i}\right)^{\Lambda_{i}}\left(z-a_{i}\right)^{E_{i}},
$$

где форма $\omega$ имеет логарифмическую особенность в $a_{i}$. (Существование такой системы следует из теоремы Племеля 9 и теоремы Биркгофа-Гротендика (1.10).) Нетрудно видеть, что набор диагональных элементов матрицы $K$ совпадает с типом расщепления расслоения $E$.

Предположим, что для некоторого $l$ имеет место неравенство $k_{l}-k_{l+1}>n-2$. Так как элементы $\omega_{m j}$ и $u_{m j}$ матричных дифференциальных форм $\omega^{\prime}$ и $\omega$ при $m \neq j$ связаны соотношением

$$
\omega_{m j}(z)=u_{m j}(z)\left(z-a_{i}\right)^{-k_{m}+k_{j}} .
$$

то для $m>l, j \leqslant l$ согласно предположению получаем $k_{j}-k_{m}>$ $n-2$. Поэтому порядки нулей дифференциальных форм $\omega_{m j}(z)$ с указанными индексами в точке $a_{i}$ больше числа $n-3$, в то время как сумма порядков полюсов в особых точках, отличных от $a_{i}$ не превосходит числа $n-1$ (так как форма $\omega^{\prime}$ имеет логарифмические особенности в этих точках). Так как форма $\omega^{\prime}$ голоморфна в бесконечности, то ее коэффициенты имеют нуль 
порядка 2 этой точке. Окончательно получаем, что для коэффициентов форм $\omega_{m j}(z)$ с указанными индексами сумма порядков нулей на сфере Римана больше числа $n-1$, в то время как сумма порядков полюсов не превосходит этого числа. Значит, эти формы тождественно равны нулю, и стало быть, форма $\omega^{\prime}$ имеет вид

$$
\omega^{\prime}=\left(\begin{array}{cc}
\omega^{1} & * \\
0 & \omega^{2}
\end{array}\right),
$$

где размер матричной формы $\omega^{1}$ равен $(l, l)$.

Но это означает, что монодромия построенной системы приводима (так как она "содержит" монодромию подсистемы с матрицей $\left.\omega^{1}\right)$, что противоречит условию. Таким образом неравенство (1.12) действительно имеет место.

Для дальнейшего нам понадобится следующая лемма, связывающая тип расщепления расслоения с показателями (т.е. с асимптотиками решений) системы в особой точке (см. [5]).

ЛЕммА 5. Для любой голоморфно обратимой в нуле матричнозначной функции $U(z)$ и для любой диагоналъной целочисленной матрицы $K$ найдется многочлен $Г(z)$ от $1 / z$ с матричными коэбфициентами, голоморфно обратимый вне точки нуль и такой, чтo

$$
\Gamma(z) z^{K} U(z)=\widetilde{U}(z) z^{D}
$$

где матрица $D=\operatorname{diag}\left(d_{1}, \ldots, d_{p}\right)$ может бъть получена из матрицы $K$ некоторой перестановкой ее диагональных элементов, $\widetilde{U}(z)$ голоморфно обратима в нуле.

Теперь мы имеем все необходимое для доказательства теоремы 2 .

ДОКАЗАТЕЛЬСтво теОРЕмЫ 2. Рассмотрим векторное расслоение $E$ из $\mathcal{F}$, построенное по неприводимому представлению $(0.4)$ и матрицам $\Lambda_{2}=\cdots=\Lambda_{n}=0, \Lambda_{1}=\operatorname{diag}\left(\lambda_{1}, \ldots, \ldots, \lambda_{p}\right)$, $\lambda_{i}-\lambda_{i+1}>(n-2)(p-1), \quad i=1, \ldots, p-1$.

Вновь рассмотрим базис мероморфных сечений расслоения $E$, голоморфный вне точки $a_{1}$ и такой, что фундаментальная матрица пространства решений системы

$$
d y=\omega y
$$


построенной по логарифмической связности $\nabla$, в окрестности точки $a_{1}$ имеет вид

$$
Y_{1}(z)=\left(z-a_{1}\right)^{-K} V(z)\left(z-a_{1}\right)^{\Lambda_{1}}\left(z-a_{1}\right)^{E_{1}},
$$

и система является фуксовой вне точки $a_{1}$.

Согласно лемме 5 существует такая матрица $\Gamma(z)$, голоморфно обратимая вне точки $a_{1}$, что

$$
\Gamma(z)\left(z-a_{1}\right)^{-K} V(z)=U(z)\left(z-a_{1}\right)^{D},
$$

где матрица $D$ получена из $-K$ некоторой перестановкой диагональных элементов, а матрица $U(z)$ голоморфно обратима в точке $a_{1}$. Так как для элементов матрицы $K$ имеют место неравенства (1.12), то для любых соседних диагональных элементов матрицы $D$ получаем $\left|d_{i}-d_{i+1}\right| \leqslant(n-2)(p-1)$. Поэтому матрица $H_{1}=D+\Lambda_{1}$ допустима, т.е. для диагональных элементов $h_{i}$ этой матрицы выполнено условие $h_{i}>h_{i+1}, i=1, \ldots, p-1$.

Перейдем от построенной системы с матрицей коэффициентов $\omega$ и фундаментальной матрицей $Y_{1}$ к системе с фундаментальной матрицей $Y_{1}^{\prime}=\Gamma(z) Y_{1}$.

Так как матрица $\Gamma(z)$ голоморфно обратима вне точки $a_{1}$, новая система будет вновь фуксовой вне $a_{1}$. В окрестности точки $a_{1}$ матрица $Y_{1}^{\prime}$ будет иметь вид

$$
\begin{aligned}
Y_{1}^{\prime} & =\Gamma(z) Y_{1}=U(z)\left(z-a_{1}\right)^{D}\left(z-a_{1}\right)^{\Lambda_{1}}\left(z-a_{1}\right)^{E_{1}} \\
& =U(z)\left(z-a_{1}\right)^{H_{1}}\left(z-a_{1}\right)^{E_{1}}
\end{aligned}
$$

с голоморфно обратимой матрицей $U(z)$, допустимой матрицей $H_{1}$ и верхнетреугольной матрицей $E_{1}$. Поэтому построенная система будет фуксовой и в точке $a_{1}$.

Различные достаточные условия реализуемости представления (0.4) фуксовой системой представлены в [5]. Приведем здесь лишь некоторые из них.

Теорема 11. Пусть все матрицы $\chi\left(\sigma_{j}\right)$ монодромии представления $\chi$ одновременно приводятся $к$ следующему виду:

$$
G_{j}=\left(\begin{array}{cc}
G_{j}^{1} & * \\
0 & G_{j}^{2}
\end{array}\right)
$$


где размер каждой матрицъ $G_{j}^{1}-(l, l)$. Пусть набор матрии, $G_{1}^{i}, \ldots, G_{n}^{i}$ определяет представление $\chi_{i}, i=1,2$, и пусть представление $\chi_{2}$ реализуется в качестве монодромии некоторой фуксовой системы. Если представление $\chi_{1}$ неприводимо и если для некоторого $i$ матрииа $G_{i}$ имеет вид

$$
G_{i}=\left(\begin{array}{cc}
G_{j}^{\prime} & * \\
0 & G_{j}^{\prime \prime}
\end{array}\right),
$$

где $G_{i}^{\prime}$ имеет размер $(t, t), 0<t \leqslant l$, то монодромия $\chi$ также может быть реализована как монодромия некоторой фуксовой системы.

ТЕОРЕма 12. Любое представление $\chi$ является подпредставлением (бакторпредставлением) представления, которое может быть реализовано в качестве монодромии некоторой фуксовой cuстемы.

ПРЕДЛОЖЕНИЕ 5. Пусть все подпредставления представления ұ неразложимы. Если матрицы монодромии этого представления можно одновременно привести к виду

$$
\chi\left(\sigma_{i}\right)=\left(\begin{array}{cccc}
\frac{G_{i}^{1}}{} & & & \\
& \underline{G_{i}^{2}} & * & \\
& 0 & \ddots & \underline{G_{i}^{m}}
\end{array}\right),
$$

так, что для некоторого $i$ матрица $\chi\left(\sigma_{i}\right)$ имеет блочный вид (1.16), где размер матрицьь $G_{i}^{\prime}$ не превосходит размера матрицы $G_{i}^{1}$, то представление х может быть реализовано как представление монодромии некоторой фуксовой системъ.

\section{$\S 1.3$. Контрпример к проблеме Римана-Гильберта}

Из теоремы 2, в частности, следует, что контрпримеры к проблеме Римана-Гильберта (если таковые имеются) следует искать среди приводимых представлений.

Рассмотрим специальный тип представлений (0.4), который в дальнейшем будем называть Б-представлениями. 
ОПРеДЕЛЕНИЕ 2. Представление (0.4) называется Б-представлением, если это представление приводимо, и если жорданова нормальная форма каждой из матриц монодромии $G_{i}$ состоит ровно из одной жордановой клетки.

Теорема 13. Б-представление (0.4) может бъть реализовано как представление монодромии некоторой фуксовой системы тогда и толъко тогда, когда тип расщепления канонического продолжения $F^{0}$ расслоения $F$, построенного по этому представлению, равен $(k, \ldots, k)$.

ДокАзАТЕльство. Если тип расщепления канонического продолжения равен $(k, \ldots, k)$, то согласно теореме 9 и соотношению (1.13) найдется система уравнений на сфере Римана с заданной монодромией и особыми точками, фуксовая в точках $a_{2}, \ldots, a_{n}$, имеющая в этих точках нулевые нормирования и такая, что ее фундаментальная матрица в окрестности точки $a_{1}$ имеет вид (1.13) с нулевой матрицей $\Lambda_{1}$ и скалярной матрицей $K=k I$ :

$$
Y_{1}(z)=\left(z-a_{1}\right)^{-k I} V(z)\left(z-a_{1}\right)^{E_{1}} .
$$

Ho

$$
\left(z-a_{1}\right)^{-k I} V(z)\left(z-a_{1}\right)^{E_{1}}=V(z)\left(z-a_{1}\right)^{-k I}\left(z-a_{1}\right)^{E_{1}} .
$$

Значит, эта система фуксова и в точке $a_{1}$. (В этом случае тривиальным оказывается расслоение, построенное из расслоения $F$ с помощью допустимых матриц $\Lambda_{1}=-k I, \Lambda_{2}=\cdots=\Lambda_{n}=0$.)

Пусть теперь Б-представление (0.4) реализовано как представление монодромии некоторой фуксовой системы (0.3) с матрицами нормирований $\Lambda_{1}, \ldots, \Lambda_{n}$. Последнее означает, что соответствующее расслоение $E \in \mathcal{F}$, построенное по данному представлению и допустимым матрицам $\Lambda_{1}, \ldots, \Lambda_{n}$, голоморфно тривиально. Пусть размерность подпредставления $\chi^{\prime}$ нашего Б-представления $\chi$ равна $l$. Обозначим через $X_{l}$ соответствующее подпространство пространства решений $X$ системы, инвариантное относительно действия монодромии $\chi$. Приведем все матрицы монодромии представления $\chi$ одновременно к блочному верхнетреугольному виду

$$
G_{i}=\left(\begin{array}{cc}
G_{i}^{\prime} & * \\
0 & G_{i}^{\prime \prime}
\end{array}\right), \quad i=1, \ldots, n,
$$


рассмотрим соответствующую фундаментальную матрицу $Y(z)$, в базисе из столбцов которой матрицы монодромии имеют указанный вид.

Так как матрица $G_{i}$ сопряжена к жордановой клетке, то согласно примеру 1 получаем, что первые $l$ элементов любого левелевского базиса пространства $X$ в любой особой точке принадлежат подпространству $X_{l}$. Поэтому согласно теореме 7 в окрестности точки $a_{i}$ матрица $Y$ представима следующим образом

$$
Y(z)=U_{i}(z)\left(z-a_{i}\right)^{\Lambda_{i}}\left(z-a_{i}\right)^{E_{i}} S_{i},
$$

где матрицы $E_{i}, S_{i}$ имеют такой же блочный верхнетреугольный вид, что и матрицы $G_{i}$, а допустимая матрица $\Lambda_{i}$ имеет вид $\Lambda_{i}=$ $\operatorname{diag}\left(\Lambda_{i}^{\prime}, \Lambda_{i}^{\prime \prime}\right)$.

Тем самым у расслоения $E$ имеется подрасслоение $F^{\prime}$ ранга $l$, построенное по представлению $\chi^{\prime}$ и допустимым матрицам $\Lambda_{i}^{\prime}$. Заметим, что степень этого подрасслоения неотрицательна, и она равна нулю тогда и только тогда, когда все матрицы $\Lambda_{i}$ скалярные: $\Lambda_{i}=c_{i} I, \quad i=1, \ldots, n$.

Действительно, пусть $\Lambda_{i}=\operatorname{diag}\left(\lambda_{i}^{1}, \ldots, \lambda_{i}^{p}\right)$. Тогда в силу допустимости имеем $\lambda_{i}^{1} \geqslant \cdots \geqslant \lambda_{i}^{p}$ и

$$
\frac{\lambda_{i}^{1}+\cdots+\lambda_{i}^{l}}{l} \geqslant \frac{\lambda_{i}^{1}+\cdots+\lambda_{i}^{p}}{p},
$$

причем равенство достигается тогда и только тогда, когда все числа $\lambda_{i}^{j}$ равны. Поэтому для степени этого подрасслоения имеем

$$
\begin{aligned}
\frac{\operatorname{deg}\left(F^{\prime}\right)}{l} & =\frac{1}{l} \sum_{i=1}^{n} \operatorname{tr}\left(\Lambda_{i}^{\prime}+E_{i}^{\prime}\right)=\sum_{i=1}^{n}\left(\rho_{i}+\frac{\lambda_{i}^{1}+\cdots+\lambda_{i}^{l}}{l}\right) \\
& \geqslant \sum_{i=1}^{n}\left(\rho_{i}+\frac{\lambda_{i}^{1}+\cdots+\lambda_{i}^{p}}{p}\right)=\frac{\operatorname{deg}(E)}{p}=0,
\end{aligned}
$$

и равенство достигается тогда и только тогда, когда все матрицы $\Lambda_{i}$ скалярные.

Но расслоение $E$ тривиально, следовательно его подрасслоение $F^{\prime}$ должно иметь неположительную степень ${ }^{37}$. Поэтому $\operatorname{deg}\left(F^{\prime}\right)=0$ и $\Lambda_{i}=c_{i} I, i=1, \ldots, n$.

\footnotetext{
${ }^{37}$ Приведенные здесь соображения могут быть проинтерпретированы на языке векторных расслоений в терминах полустабильности векторного расслоения со связностъю, см. Bolibrukh A. On sufficient conditions for the existence of a Fuchsian equation with prescribed monodromy // J. Dynam. Control Systems, 1999, 5(4), 453-472.
} 
Преобразуем построенную систему к системе с фундаментальной матрицей

$$
Y^{\prime}(z)=\left(\prod_{i=2}^{n}\left(z-a_{i}\right)^{-c_{i}}\left(z-a_{1}\right)^{c}\right) Y(z), \quad c=\sum_{i=2}^{n} c_{i} .
$$

Эта система будет по-прежнему фуксовой во всех точках и иметь нулевые нормирования в точках $a_{2}, \ldots, a_{n}$. В точке $a_{1}$ согласно построению фундаментальная матрица этой системы может быть представлена в виде $(1.17)$ с $k=-\left(c+c_{1}\right)$. Но последнее означает, что тип расщепления канонического продолжения $F^{0}$ расслоения, построенного по исходному Б-представлению, равен $(k, \ldots, k)$.

СлЕДСТвИЕ 3. Если Б-представление может быть реализовано как представление монодромии некоторой фуксовой системы, то степень канонического продолжсения $F^{0}$ расслоения $F$, построенного по этому представлению, должна делиться начело на ранг представления.

Оказывается, существуют Б-представления, которые этому свойству не удовлетворяют.

Следующий пример является контрпримером к утверждению Гильберта. Он означает, что проблема Римана-Гильберта имеет в общем случае отрицательное решение.

ПримеР 2. Рассмотрим матрицы

$$
\begin{gathered}
G_{1}=\left(\begin{array}{llll}
1 & 1 & 0 & 0 \\
0 & 1 & 1 & 0 \\
0 & 0 & 1 & 1 \\
0 & 0 & 0 & 1
\end{array}\right), G_{2}=\left(\begin{array}{rrrrr}
3 & 1 & 1 & -1 \\
-4 & -1 & 1 & 2 \\
0 & 0 & 3 & 1 \\
0 & 0 & -4 & -1
\end{array}\right), \\
G_{3}=\left(\begin{array}{rrrr}
-1 & 0 & 2 & -1 \\
4 & -1 & 0 & 1 \\
0 & 0 & -1 & 0 \\
0 & 0 & 4 & -1
\end{array}\right)
\end{gathered}
$$

и произвольный набор точек $a_{1}, a_{2}, a_{3}$. Представление $\chi$ с особыми точками $a_{1}, a_{2}, a_{3}$ и матрицами монодромии $G_{i}, i=1,2,3$, не может быть реализовано в качестве представления монодромии какой-либо фуксовой системы. 
ДокАЗАтЕЛьСтво. Заметим, что $G_{1} \cdot G_{2} \cdot G_{3}=I$, матрица $G_{2}$ может быть преобразована к матрице $G_{1}$, а матрица $G_{3}$ может быть преобразована к жордановой клетке с собственным значением -1 . Действительно, для матрицы $G_{2}$ имеем

$$
S_{2}^{-1} G_{2} S_{2}=\left(\begin{array}{llll}
1 & 1 & 0 & 0 \\
0 & 1 & 1 & 0 \\
0 & 0 & 1 & 1 \\
0 & 0 & 0 & 1
\end{array}\right), \quad S_{2}=\frac{1}{3}\left(\begin{array}{rrrr}
3 & 0 & 0 & 0 \\
-6 & 3 & -3 & 4 \\
0 & 0 & 1 & -1 \\
0 & 0 & -2 & 3
\end{array}\right),
$$

а для матрицы $G_{3}$ получаем

$$
\begin{gathered}
S_{3}^{-1} G_{3} S_{3}=\left(\begin{array}{rrrr}
-1 & 1 & 0 & 0 \\
0 & -1 & 1 & 0 \\
0 & 0 & -1 & 1 \\
0 & 0 & 0 & -1
\end{array}\right), \\
S_{3}=\frac{1}{64}\left(\begin{array}{rrrr}
0 & 16 & 4 & 3 \\
64 & 0 & 0 & 0 \\
0 & 0 & 0 & -4 \\
0 & 0 & -16 & -12
\end{array}\right) .
\end{gathered}
$$

Степень канонического продолжения равна

$$
\operatorname{deg}\left(F^{0}\right)=\operatorname{tr} E_{1}+\operatorname{tr} E_{2}+\operatorname{tr} E_{3}=0+0+\frac{1}{2} \cdot 4=2 \not \equiv 0 \quad(\bmod 4) .
$$

Поэтому согласно следствию 3 это представление не может быть реализовано как представление монодромии какой-либо фуксовой системы.

Оказывается, в размерности три все контрпримеры к проблеме Римана-Гильберта даются Б-представлениями. А именно имеет место следующая теорема [1]

Теорема 14. Проблема Римана-Гилъберта для представления (0.4) размерности $p=3$ имеет положительное решение тогда и толъко тогда, когда это представление является Б-представлением и тип расщепления канонического продолжения расслоения, построенного по этому представлению, равен $(k, \ldots, k)$. 
Минимальная размерность представления, в которой появляется контрпример к проблеме Римана-Гильберта, равна трем, а минимальное число особых точек при этом равно четырем (см. [1]).

Рассмотрим систему

$$
\begin{gathered}
d f=\left(\left(\begin{array}{ccc}
0 & 1 & 0 \\
0 & z & 0 \\
0 & 0 & -z
\end{array}\right) \frac{d z}{z^{2}}+\frac{1}{6}\left(\begin{array}{ccc}
0 & 6 & 0 \\
0 & -1 & 1 \\
0 & -1 & 1
\end{array}\right) \frac{d z}{z+1}\right. \\
\left.+\frac{1}{2}\left(\begin{array}{ccc}
0 & 0 & 2 \\
0 & -1 & -1 \\
0 & 1 & 1
\end{array}\right) \frac{d z}{z-1}+\frac{1}{3}\left(\begin{array}{ccc}
0 & -3 & -3 \\
0 & -1 & 1 \\
0 & -1 & 1
\end{array}\right) \frac{d z}{z-1 / 2}\right) f .
\end{gathered}
$$

Эта система фуксова в точках $-1,1,1 / 2$, а точка $\infty$ является для нее точкой голоморфности, так как вычет в бесконечности формы системы равен нулю. Нетрудно показать, что точка 0 является регулярной особой точкой для этой системы.

Также непосредственно проверяется, что все матрицы монодромии этой системы приводятся к жордановой клетке с собственным значением 1 (каждая матрица с помощью своего преобразования). Так как эта система содержит одномерную подсистему, монодромия всей системы приводима. Значит, представление монодромии этой системы является Б-представлением.

Показатели этой системы в точках $-1,1,1 / 2$ равны нулю, а в точке 0 ее фундаментальная матрица может быть записана в виде (см. [1], [5])

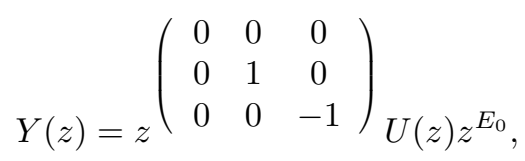

где $U(z)$ голоморфно обратимая матрица в точке нуль.

Отсюда немедленно следует, что тип расщепления канонического продолжения расслоения, построенного по представлению монодромии этой системы, равен $(1,0,-1)$, и стало быть, согласно теореме 13 это представление не может быть реализовано никакой фуксовой системой.

Построенный контрпример обладает следующим свойством неустойчивости. При любом сколь угодно малом изменении положения особой точки ноль и при сохранении матриц монодромии 
соответствующее представление монодромии уже может быть реализовано как представление монодромии некоторой фуксовой системы. Поэтому этот пример представляет интерес для теории изомонодромных деформаций фуксовых систем. А именно, точка $(0,-1,1,1 / 2)$ пространства $\mathbb{C}^{4}$ является подвижной особой точкой для соответствующего уравнения Шлезингера изомонодромных деформаций, построенного по этому представлению ${ }^{38}$.

Исходя из приведенного примера, нетрудно построить контрпример к проблеме Римана-Гильберта для любого числа особых точек, большего трех и для любого представления (0.4) ранга, большего двух (и тем самым доказать теорему 1) (см. [1]).

Рассмотрим множество $\mathcal{F}$ расслоений, построенных в главе 1 по неприводимому представлению (0.4).

Для произвольного расслоения $F \in \mathcal{F}$ рассмотрим число

$$
\gamma(F)=p k_{1}-\operatorname{deg}(F)=p k_{1}-\sum_{i=1}^{p} k_{i},
$$

где числа $k_{1}, \ldots, k_{p}$ задают тип расщепления расслоения $F$.

ОПРЕДЕЛЕНИЕ 3. ЧИсЛО

$$
\gamma_{m}(\chi)=\sup _{F \in \mathcal{F}} \gamma(F)
$$

назовем максимальным фуксовым весом представления $\chi$.

Это число согласно теореме 10 не превосходит числа $(n-2) \times$ $p(p-1) / 2$. Оно оказывается тесно связанным с асимптотиками решений фуксовых систем с данной монодромией.

Рассмотрим пространство $X$ решений фуксовой системы $(0.1),(0.3)$ двух уравнений. Обозначим показатели этой системы в точке $a_{i}$ через $\beta_{i}^{1}, \beta_{i}^{2}$ и рассмотрим число

$$
\gamma(\omega)=\sum_{i=1}^{n}\left|\beta_{i}^{1}-\beta_{i}^{2}\right|,
$$

которое имеет смысл суммы разностей асимптотик решений по всем особым точкам.

Рассмотрим множество $\Omega$ всех фуксовых систем двух уравнений на $\overline{\mathbb{C}}$ с данным представлением (0.4) монодромии (согласно теореме 2 это множество не пусто).

\footnotetext{
${ }^{38}$ Bolibruch A. On orders of movable poles of the Schlesinger equation //
} J. Dynam. Control Systems, 2000, 6(1), 57-74. 
Teopema 15.

$$
\min _{\omega \in \Omega} \gamma(\omega)=\gamma_{m}(\chi) .
$$

В терминах минимального фуксова веса представления удается выразить минимально возможное число дополнительных ложных особых точек $m^{0}$ (посчитанных с кратностями, которые совпадают с порядками нулей вронскиана соответствующего "минимального" фуксового уравнения), возникающих при построении скалярного фуксового дифференциального уравнения с неприводимой монодромией (0.4).

Теорема 16. Минимально возможное число $m^{0}$ дополнительных ложных особых точек, возникающих при построении скалярного фуксового дифференииального уравнения с неприводимой монодромией (0.4), равно

$$
m^{0}=\frac{(n-2) p(p-1)}{2}-\gamma_{m}(\chi) .
$$

Для теории изомонодромных деформаций особый интерес представляет следующая проблема, которую можно рассматривать как некоторое обобщение проблемы Римана-Гильберта.

Пусть заданы неприводимое представление (0.4) и набор $\Lambda$ целочисленных матрии, $\Lambda_{1}, \ldots, \Lambda_{n}$, удовлетворяющие условию

$$
\sum=\sum_{i=1}^{n} \sum_{j=1}^{p} \lambda_{i}^{j}=0 .
$$

Всегда ли найдется фуксова система с данными (0.4) и $\Lambda$ ? (Задача существования фуксовой системы с заданными асимптотиками решений и монодромией.)

Ответ на этот вопрос в общем случае отрицателен (см. [1]). Имеются наборы нормирований $\Lambda$, которые не реализуются никакими фуксовыми системами с данной неприводимой монодромией. Будем называть такие наборы (для данной монодромии и особых точек) запрещенными наборами нормирований.

Ясно, что если набор $\Lambda=\left\{\Lambda_{1}, \ldots, \Lambda_{n}\right\}$ запрещен, то то же самой верно для набора $\Lambda^{\prime}=\left\{\Lambda_{1}^{\prime}, \ldots, \Lambda_{n}^{\prime}\right\}$, где

$$
\Lambda_{i}^{\prime}=\Lambda_{i}+k_{i} I, \quad i=1, \ldots, n, \quad k_{i} \in \mathbb{Z}, \quad \sum_{i=1}^{n} k_{i}=0 .
$$


(В самом деле, преобразование

$$
y^{\prime}=\Gamma y, \quad \Gamma(z)=\prod_{i=1}^{n}\left(z-a_{i}\right)^{\Lambda_{i}^{\prime}-\Lambda_{i}}
$$

переводит исходную фуксову систему с набором нормирований $\Lambda$ в фуксову систему с набором нормирований $\Lambda^{\prime}$.)

Будем называть такие наборы нормирований эквивалентными.

Верно ли, что для каждого неприводимого представления (0.4) существует лишь конечное число неэквивалентных запрещенных наборов нормирований?

Если бы сформулированная гипотеза была верна, то это упростило бы исследование некоторых вопросов теории фуксовых уравнений. Например, удалось бы получить классификацию четырехмерных представлений, нереализуемых никакими фуксовыми системами и т.д.

Та же самая гипотеза может быть сформулирована в терминах векторных расслоений. Рассмотрим множество векторных расслоений $\mathcal{F}$ на сфере Римана с логарифмическими связностям, построенное по данному неприводимому представлению (0.4) (см. главу 1$)$. Обозначим через $\mathcal{F}_{0}$ подмножество топологически тривиальных расслоений (т.е., расслоений, степень которых равна нулю) в $\mathcal{F}$. Хорошо известно, что пространство аналитически нетривиальных векторных расслоений со степенью равной нулю образует аналитическое подмножество $N$ коразмерности один в пространстве всех аналитических векторных расслоений с тем же условием на степень ${ }^{39}$. Верно ли, что пересечение $\mathcal{F}_{0}$ с $N$ состоит из конечного числа точек? Это другая, геометрическая формулировка гипотезы о запрещенных нормированиях.

В работах [8], [10] показано, что эта гипотеза неверна и приведены соответствующие классы неприводимых представлений с бесконечным числом запрещенных нормирований. Строятся они следующим образом.

Обозначим через $\Sigma$ множество точек $\left(a_{1}, \ldots, a_{n}\right)$, а через $\Sigma^{\prime}$ множество $\left(b_{1}, \ldots, b_{k}\right)$. Рассмотрим рациональное отображение

$$
f:(\overline{\mathbb{C}}, \Sigma) \longrightarrow\left(\overline{\mathbb{C}}, \Sigma^{\prime}\right), \quad f=\frac{R(z)}{Q(z)},
$$

${ }^{39}$ Bojarski B. Connections between complex and global analysis // Complex analysis. Methods, trends, and applications. Berlin: Akad. Verl., 1983. 97-110; Прессли Э., Сигал Г. Группы петель. М.: Мир, 1990. 
такое, что многочлены $R$ и $Q$ не имеют общих делителей, $f^{-1}\left(\Sigma^{\prime}\right)=\Sigma$ и по крайней мере одна из точек множества $\Sigma$ является критической точкой для $f$ (т.е. $d f=0$ в этой точке).

Пусть представление

$$
\chi^{\prime}: \pi_{1}\left(\overline{\mathbb{C}} \backslash \Sigma^{\prime}, t_{0}\right) \longrightarrow G L(p, \mathbb{C})
$$

неприводимо. Рассмотрим представление

$$
\chi_{b}: \pi_{1}\left(\overline{\mathbb{C}} \backslash \Sigma, z_{0}\right) \longrightarrow G L(p, \mathbb{C}),
$$

где $\chi_{b}=\chi^{\prime} \circ f_{\#}, \quad f\left(z_{0}\right)=t_{0}$.

Теорема 17. Для представления $\chi_{b}$ существует бесконечное число запрещенных неэквивалентных наборов нормирований.

В случае, когда показатели во всех точках кроме одной зафиксированы, данная теорема дает полное описание всех неприводимых представлений с бесконечным числом запрещенных нормирований ${ }^{40}$.

В общем случае вопрос о том, все ли представления с бесконечным числом запрещенных нормирований даются теоремой 17 , остается открытым.

В работе [8] представлены также новые серии контрпримеров, не сводящиеся к Б-представлениям.

\section{2. Биркгофова стандартная форма}

Эта задача, которая на первый взгляд выглядит как локальная (и исходная система, и искомое преобразование определены лишь локально в окрестности точки $\infty$ ), на самом деле носит глобальный характер, так как итоговая система задана уже на всей сфере Римана. Поэтому ее естественно переформулировать в терминах расслоений и связностей.

Рассмотрим фундаментальную матрицу $Y(z)$ системы $(0.7)$, в базисе из столбцов которой матрица $G$ монодромии системы имеет верхний треугольный вид. Тогда

$$
Y(z)=T(z) z^{E}
$$

\footnotetext{
${ }^{40}$ Kostov V.P. Quantum states of monodromy groups // J. Dynam. Control Systems, 1998, 5(1), 51-100; [10].
} 
где матричная функция $T(z)$ однозначна и голоморфно обратима в $O_{\infty}$, а $E=\frac{1}{2 \pi i} \ln G$, причем собственные значения $\rho^{j}$ матрицы $E$ удовлетворяют неравенствам (1.1).

Зададим расслоение $F$ с помощью следующего координатного описания: $F=\left(O_{\infty}, O_{0}=\mathbb{C}, g_{\infty 0}=T(z)\right)$. Формы

$$
\omega_{\infty}=\frac{C(z)}{z} d z \quad \text { и } \quad \omega_{0}=\frac{E}{z} d z,
$$

определенные в окрестностях $O_{\infty}$ и $O_{0}$ соответственно, задают в расслоении $F$ связность $\nabla$, голоморфную вне точек $0, \infty$, имеющую логарифмическую особенность в нуле и полюс порядка $r+1$ в бесконечности.

Действительно, из (0.7) следует, что

$$
\frac{C(z)}{z} d z=d Y(z)(Y(z))^{-1}=d T(z)(T(z))^{-1}+T(z) \frac{E}{z} d z(T(z))^{-1}
$$

и следовательно, $\omega_{\infty}=d g_{\infty 0} g_{\infty 0}^{-1}+g_{\infty 0} \omega_{0} g_{\infty 0}^{-1}$.

Рассмотрим базис $(e)$ из $p$ сечений расслоения $F$, линейно независимых и голоморфных вне точки ноль и мероморфных в этой точке. В базисе из этих сечений форма $(\widetilde{C}(z) / z) d z$ связности $\nabla$ имеет вид

$$
\widetilde{C}(z)=\widetilde{C}_{r} z^{r}+\cdots+\widetilde{C}_{0}+\frac{\widetilde{C}_{1}}{z}+\cdots+\frac{\widetilde{C}_{-k}}{z^{k}} .
$$

Действительно, на координатном языке последнее утверждение означает существование такой голоморфно обратимой в окрестности $O_{\infty}$ матрицы $\Gamma$ и такой голоморфно обратимой в $\mathbb{C} \backslash 0$ и мероморфной в нуле матричной функции $U(z)$, что $\Gamma g_{\infty 0}=U(z)$ (здесь столбцы матриц $\Gamma$ и $U$ задают координатное описание элементов базиса $(e))$. Поэтому система линейных дифференциальных уравнений (0.10) с фундаментальной матрицей

$$
\widetilde{Y}(z)=\Gamma(z) Y(z)=\Gamma(z) T(z) z^{E}=U(z) z^{E},
$$

определенной во всей комплексной плоскости $\mathbb{C}$, и с матрицей коэффициентов (2.2), не имеет особых точек на сфере Римана, за исключением точек ноль и бесконечность, причем точка ноль является для нее регулярной особой точкой. Поэтому матрица $\widetilde{C}(z)$ является рациональной функцией с единственным полюсом в нуле в комплексной плоскости и с полюсом порядка $r$ в бесконечности.

Тем самым мы доказали следующее утверждение. 
Теорема 18. Любая система (0.7) с помощью аналитического преобразования может быть приведена к системе (0.10) на сфере Римана с матрицей коэффичиентов $\widetilde{C}(z)$ вида (2.2). Причем особая точка ноль будет для этой системы регулярной особой точкой.

Теорема 18 означает, что, решая задачу о биркгофовой стандартной форме, мы с самого начала можем рассматривать вместо системы (0.7) систему (0.10) с матрицей коэффициентов вида $(2.2)$, для которой точка ноль является регулярной особой точкой.

Приведем матрицу монодромии $G$ с помощью матрицы $S$ к верхнетреугольному виду (возможно, отличному от того, к которому она была приведена в разложении (2.1)) и рассмотрим произвольную допустимую матрицу $\Lambda=\operatorname{diag}\left(\lambda_{1}, \ldots, \lambda_{p}\right)$, т.е. матрицу с целочисленными диагональными элементами, удовлетворяющими неравенствам $\lambda_{1} \geqslant \cdots \geqslant \lambda_{p}$.

Зададим расслоение $F^{\Lambda}$ с помощью следующего координатного описания: $F^{\Lambda}=\left(O_{\infty}, O_{0}=\mathbb{C}, g_{\infty 0}^{\Lambda}=T(z) z^{-\Lambda}\right)$. Формы

$$
\omega_{\infty}=\frac{C(z)}{z} d z \quad \text { и } \quad \omega_{0}^{\Lambda}=\left(\Lambda+z^{\Lambda} E z^{-\Lambda}\right) \frac{d z}{z},
$$

определенные в окрестностях $O_{\infty}$ и $O_{0}$ соответственно, задают в расслоении $F^{\Lambda}$ связность $\nabla^{\Lambda}$, голоморфную вне точек $0, \infty$, имеющую логарифмическую особенность в нуле и полюс порядка $r+1$ в бесконечности.

Голоморфный тип построенного расслоения зависит от матрицы $\Lambda$ и от способа приведения матрицы монодромии к верхнетреугольному виду (т.е. от матрицы $S$ ).

Обозначим множество построенных расслоений со связностями через $\mathcal{E}$.

Следующее утверждение аналогично теореме 8 и доказывается почти также (с некоторыми упрощениями, так как приходится следить всего за одной фуксовой точкой).

Теорема 19. Система (0.7) может быть приведена аналитическим преобразованием $к$ биркгофовой стандартной форме тогда и только тогда, когда множество $\mathcal{E}$ содержит хотя бы одно голоморфно тривиальное расслоение. 
Имеет место в этом случае и аналог теоремы 10.

Теорема 20. Рассмотрим расслоение $E \in \mathcal{E}$ со связностъю $\nabla$, построенное по неприводимой системе (0.7). Для типа расщепления этого расслоения имеют место следующие неравенства

$$
k_{i}-k_{i+1} \leqslant r, \quad i=1, \ldots, p-1
$$

ДокАзАтЕЛьство. Согласно теореме Биркгофа-Гротендика (см. $(1.10))$ найдутся такие матрицы $\Gamma \in H^{0}\left(O_{\infty}\right), U \in H^{0}(\mathbb{C})$, что

$$
\Gamma g_{\infty 0}^{\Lambda}(z)=\Gamma T(z) z^{-\Lambda}=z^{-K} U(z)
$$

где диагональные элементы матрицы $K$ задают тип расщепления расслоения $E$ (см. формулу (1.13)).

Аналитическое преобразование исходной системы, задаваемое матрицей $\Gamma$, переводит ее в систему (0.10), где

$$
\begin{gathered}
\frac{\widetilde{C}(z)}{z} d z=-\frac{K}{z} d z+z^{-K} \omega z^{K} \\
\omega=d U U^{-1}+U\left(\Lambda+z^{\Lambda} E z^{-\Lambda}\right) U^{-1} \frac{d z}{z},
\end{gathered}
$$

а фундаментальная матрица пространства решений системы (0.10) в $\mathbb{C}$ представляется в виде

$$
Y^{\prime}(z)=z^{-K} U(z) z^{\Lambda} z^{E}
$$

Заметим, что форма $\omega$ имеет логарифмическую особенность B 0 .

Предположим, что для некоторого $l$ имеет место неравенство $k_{l}-k_{l+1}>r$. Так как элементы $\omega_{i j}^{\prime}$ и $\omega_{i j}$ матричных дифференциальных форм $\widetilde{C}(z) d z / z$ и $\omega$ связаны соотношением

$$
\omega_{i j}^{\prime}(z)=\omega_{i j}(z) z^{-k_{i}+k_{j}} .
$$

то для $i>l, j \leqslant l$ согласно предположению получаем $k_{j}-k_{i}>r$. Поэтому порядки нулей дифференциальных форм $\omega_{i j}^{\prime}(z)$ с указанными индексами в точке 0 больше числа $r-1$, в то время как порядки полюсов коэффициентов этих форм в бесконечности не 
превосходят числа $r-1$ (так как форма $\widetilde{C}(z) d z / z$ имеет полюс порядка $r+1$ в $\infty$ ). Значит, эти формы тождественно равны нулю, и стало быть, форма $\widetilde{C}(z) d z / z$ имеет вид $(0.13)$. Значит, исходная система приводима. Полученное противоречие означает, что неравенства (2.3) имеют место.

Теперь мы имеем все необходимое для доказательства следующего утверждения, которое аналогично теореме 2 .

ТЕОрема 21. Любая неприводимая система (0.7) может быть преобразована $к$ биркгофовой стандартной форме с помощъю аналитического преобразования (0.9).

ДоказАтельство. Рассмотрим расслоение $F \in \mathcal{E}$, построенное по допустимой матрице $\Lambda$, удовлетворяющей условию $\lambda_{i}-\lambda_{i+1}>$ $r(p-1), \quad i=1, \ldots, p-1$.

Действуя так же, как при доказательстве предыдущего утверждения, приведем исходную систему с помощью аналитического преобразования к виду (0.10) с фундаментальной матрицей (2.4).

Согласно лемме 5 существует такая матрица $\Gamma^{\prime}(z)$, голоморфно обратимая вне точки 0 , что

$$
\Gamma^{\prime}(z) z^{-K} U(z)=U^{\prime \prime}(z) z^{D}
$$

где матрица $D$ получена из $-K$ некоторой перестановкой диагональных элементов, а матрица $U^{\prime \prime}(z)$ голоморфно обратима в $\mathbb{C}$. Так как для элементов матрицы $K$ имеют место неравенства (2.3), то для любых соседних диагональных элементов матрицы $D$ получаем $\left|d_{i}-d_{i+1}\right| \leqslant r(p-1)$. Поэтому матрица $H=D+\Lambda$ допустима, т.е. для диагональных элементов $h_{i}$ этой матрицы выполнено условие $h_{i} \geqslant h_{i+1}, i=1, \ldots, p-1$.

Перейдем от системы $(0.10)$ к системе с матрицей коэффициентов $C^{\prime \prime}(z)$ и фундаментальной матрицей $Y^{\prime \prime}$ с помощью аналитической в $\overline{\mathbb{C}} \backslash 0$ замены $Y^{\prime \prime}=\Gamma^{\prime}(z) Y^{\prime}$. Тогда

$$
Y^{\prime \prime}(z)=U^{\prime \prime}(z) z^{H} z^{E}
$$

с голоморфно обратимой матрицей $U^{\prime \prime}(z)$, т.е. построенная система фуксова в нуле. Согласно построению эта система не имеет 
особых точек кроме точек 0 и $\infty$, значит, матрица $C^{\prime \prime}(z)$ коэффициентов этой системы голоморфна в $\mathbb{C}$ (напомним, что система имеет вид (0.7)).

В силу аналитичности проведенных замен, матрица $C^{\prime \prime}(z)$ коэффициентов этой системы имеет полюс того же порядка $r$ в бесконечности, что и матрица исходной системы (0.7). По теореме Лиувилля получаем, что $C^{\prime \prime}(z)$ - матричный многочлен степени $r$ от $z$.

\section{3. Изомонодромные деформации фуксовых систем}

Рассмотрим семейство фуксовых систем

$$
\frac{d y}{d z}=\left(\sum_{i=1}^{n} \frac{B_{i}(a)}{z-a_{i}}\right) y, \quad \sum_{i=1}^{n} B_{i}(a)=0,
$$

голоморфно зависящее от параметра $a=\left(a_{1}, \ldots, a_{n}\right) \in D\left(a^{0}\right)$, где $D\left(a^{0}\right)$ - шар малого радиуса с центром в точке $a^{0}=\left(a_{1}^{0}, \ldots, a_{n}^{0}\right)$ пространства $\mathbb{C}^{n} \backslash \bigcup_{i \neq j}\left\{\left(a_{i}-a_{j}\right)=0\right\}$.

Семейство (3.1) задано на пространстве $T=\overline{\mathbb{C}} \times D\left(a^{0}\right) \backslash$ $\bigcup_{i=1}^{n}\left\{\left(z-a_{i}\right)=0\right\}$, которое ретрагируется (при помощи некоторого отображения $r$ ) на $\overline{\mathbb{C}} \backslash\left\{a_{1}^{0}, \ldots, a_{n}^{0}\right\}$. Поэтому фундаментальная группа $\pi_{1}\left(T,\left(z_{0}, a^{0}\right)\right)$ изоморфна группе $\pi_{1}\left(\overline{\mathbb{C}} \backslash\left\{a_{1}^{0}, \ldots, a_{n}^{0}\right\}, z_{0}\right)$, которая порождена гомотопическими классами петель $g_{i}^{0}$, обходящими особые точки $a_{i}^{0}$ соответственно по окружностям малого радиуса. Мы будем обозначать гомотопический класс петли $g_{i}^{0}$ по-прежнему через $g_{i}^{0}$.

Семейство (3.1) называется изомонодромным (или изомонодромной деформачией исходной фуксовой системы, соответствующей значению параметра $a=a^{0}$ ), если для любого фиксированного $а$ соответствующая система из (3.1) имеет ту же самую монодромию, что и при $a=a^{0}$ (по отношению к гомотопическим классам петель $g_{i}^{a}=r_{\#}^{-1}\left(g_{i}^{0}\right)$ и $g_{i}^{0}$ соответственно). Последнее означает, что для каждого значения параметра $a$ найдется фундаментальная матрица $Y(z, a)$ соответствующей системы из (3.1), имеющая одни и те же матрицы монодромии 
(по отношению к $g_{i}^{a}$ ) для всех $a \in D\left(a^{0}\right)$. В этом случае семейство матриц $Y(z, a)$ называется изомонодромным семейством матрич.

Нетрудно показать, см. ${ }^{41}$ и [9], что

ПРЕДЛОЖЕНИЕ 6. Для любого изомонодромного семейства (3.1) найдется изомонодромное семейство фундаментальных матрии, аналитическое по совокупности z и а в $T=\overline{\mathbb{C}} \times D\left(a^{0}\right) \backslash$ $\bigcup_{i=1}^{n}\left\{\left(z-a_{i}\right)=0\right\}$.

Матрица $Y(z, a)$ как функция на $T$ имеет некоторую монодромию, которая в силу аналитичности $Y(z, a)$ зависит лишь от гомотопических классов петель в $T$ с началом в $\left(z_{0}, a^{0}\right)$. Из условия изомонодромности этой матрицы следует, что ее матрицы монодромии как функции начальной точки $\left(z_{0}, a^{0}\right)$ при фиксированном $z_{0}$ являются локально постоянными по отношению к изменению $a^{0}$. С другой стороны, из определения монодромии системы линейных дифференциальных уравнений следует, что эти матрицы локально постоянны по отношению к изменению $z_{0}$ при любом фиксированном $a^{0}$.

Следовательно, матричная дифференциальная 1-форма $\omega=$ $d Y(z, a) Y^{-1}(z, a)$ является однозначной и может быть рассмотрена как форма на $T$. Действительно, для всех $g \in \pi_{1}\left(T,\left(z_{0}, a^{0}\right)\right)$ имеем для аналитического продолжения $g^{*} \omega$ формы $\omega$ вдоль $g$

$$
g^{*} \omega=d g^{*} Y(z, a) g^{*} Y^{-1}(z, a)=(d Y(z, a)) G_{g} G_{g}^{-1} Y^{-1}(z, a)=\omega .
$$

По построению пфаффова система

$$
d y=\omega y
$$

на $T$ вполне интегрируема (это означает, что $d \omega=\omega \wedge \omega$ ) и для любого фиксированного значения $a$ она совпадает с соответствующей фуксовой системой из (3.1). В результате получаем следующее утверждение ${ }^{42}$.

${ }^{41}$ Anosov D. V. Concerning the definition of isomonodromic deformation of Fuchsian systems // Ulmer Seminaire über Funktionalysis und Differentialgleichungen. Heft 2, 1997, 1-12.

${ }^{42}$ Cм. также Iwasaki K. et al. From Gauss to Painlevè. A modern theory of special functions. Braunschweig: Vieweg, 1991. 
Теорема 22. Семейство (3.1) фуксовых систем является изомонодромным тогда и только тогда, когда на $T$ существует матричная дифференциальная 1-форма $\omega$ такая, что

i) $\omega=\sum_{i=1}^{n} \frac{B_{i}(a)}{z-a_{i}} d z$ для любого фиксированного $a \in D\left(a^{0}\right)$;

ii) $d \omega=\omega \wedge \omega$.

Отсюда следует, что любое изомонодромное семейство (3.1) полностью определяется соответствующей формой $\omega$ со свойствами i), ii). Нашей целью является описание общего вида такой формы $\omega$.

Наиболее известный вид изомонодромных деформаций задается формой

$$
\omega_{s}=\sum_{i=1}^{n} \frac{B_{i}(a)}{z-a_{i}} d\left(z-a_{i}\right) .
$$

Соответствующая деформация называется деформацией Шлезингера. Непосредственное вычисление показывает, что условие іi) для этой формы эквивалентно следующему соотношению:

$$
d B_{i}(a)=-\sum_{j=1, j \neq i}^{n} \frac{\left[B_{i}(a), B_{j}(a)\right]}{a_{i}-a_{j}} d\left(a_{i}-a_{j}\right),
$$

которое называется уравнением Шлезингера.

Для фундаментальной матрицы $Y^{s}(z, a)$ пфаффовой системы (3.2) с формой $\omega=\omega_{s}$ имеет место следующее тождество:

$$
Y^{s}(\infty, a) \equiv \text { const. }
$$

Действительно,

$$
d_{a} Y^{s}(\infty, a)\left(Y^{s}(\infty, a)\right)^{-1}=-\left.\sum_{i=1}^{n} \frac{B_{i}(a)}{z-a_{i}} d\left(a_{i}\right)\right|_{z=\infty} \equiv 0 .
$$

Поэтому тождество (3.5) действительно имеет место.

Произвольные изомонодромные деформации не сводятся к одним лишь деформациям Шлезингера (3.3). В самом деле, для произвольного изомонодромного семейства (3.1) вида (3.3) можно рассмотреть семейство с изомонодромной фундаментальной матрицей $Y^{\prime}(z, a)=\Gamma(a) \times Y(z, a)$, где $\Gamma\left(a^{0}\right)=I, \Gamma(a) \neq$ const. 
Новое семейство вновь будет изомонодромным, но описывающая его форма будет иметь вид

$$
\omega=\sum_{i=1}^{n} \frac{B_{i}^{\prime}(a)}{z-a_{i}} d\left(z-a_{i}\right)+\sum_{r=1}^{n} \gamma_{r}(a) d a_{r},
$$

где $B_{i}^{\prime}=\Gamma B_{i} \Gamma^{-1}, \sum_{r=1}^{n} \gamma_{r} d a_{r}=d \Gamma \Gamma^{-1}$. Ясно, что для изомонодромной фундаментальной матрицы этого семейства нормализующее условие (3.5) не будет выполнено.

Но деформации $(3.3),(3.6)$ не исчерпывают все возможные виды деформаций в резонансном случае (хотя они полностью описывают все такие деформации в случае отсутствия резонансов ${ }^{43}$ ).

Прежде чем переходить к формулировке основной теоремы этой главы, рассмотрим следующее важное утверждение, относящееся к изомонодромным фуксовым семействам, которое по сути является теоремой Гантмахера о виде решений фуксовой системы с параметром.

Рассмотрим аналитическую изомонодромную фундаментальную матрицу $Y(z, a)$, матрицы монодромии $G_{i}$ которой имеют блочный диагональный вид $G_{i}=\operatorname{diag}\left(G_{i}^{1}, \ldots, G_{i}^{k}\right)$, где каждый блок $G_{i}^{j}$ соответствует некоторому корневому подпространству размерности $s_{j}$ оператора монодромии. Обозначим через $E_{i}=$ $\operatorname{diag}\left(E_{i}^{1}, \ldots, E_{i}^{k}\right)$ матрицу $(2 \pi i)^{-1} \ln G_{i}$, собственные значения $\rho_{i}^{j}$ которой нормализованы согласно (1.1).

Теорема 23. Для фундаменталъной матрицы $Y(z, a)$ семейства (3.1) имеет место следующее разложение в окрестности $\left\{z-a_{i}=0\right\}$ :

$$
Y(z, a) S(a)=U_{i}(z, a)\left(z-a_{i}\right)^{\Lambda_{i}}\left(z-a_{i}\right)^{E_{i}(a)},
$$

где $S(a)$ голоморфно обратима в $D\left(a^{0}\right)$ и имеет тот же самый блочно-диагональный вид, что и $E_{i}, U_{i}(z, a)$ голоморфно обратима в окрестности $\left\{z-a_{i}=0\right\}, \Lambda_{i}$ имеет тот же самый блочнодиагональный вид $\Lambda_{i}=\operatorname{diag}\left(\Lambda_{i}^{1}, \ldots, \Lambda_{i}^{k}\right)$, что и $E_{i}$, с целочисленными диагональными матрицами $\Lambda_{i}^{j}$, элементы ${ }^{s} \lambda_{i}^{j}$ которых удовлетворяют следующим неравенствам:

$$
{ }^{s} \lambda_{i}^{j} \geqslant{ }^{s+1} \lambda_{i}^{j},
$$

$u E_{i}(a)=S^{-1}(a) E_{i} S(a)$.

\footnotetext{
${ }^{43}$ Ibid.
} 
ДокАЗАтЕльство. Согласно теореме Гантмахера ${ }^{44}$ (см. также [1] или [5]) для каждого фиксированного $a \in D\left(a^{0}\right)$ существует матрица $S(a)$ такая, что разложение (3.7) имеет место с некоторой матрицей $\Lambda_{i}(a)$ (Если матрица монодромии имеет единственно собственное значение, то это разложение совпадает с (1.6)). Вначале мы докажем, что матричная функция $\Lambda_{i}(a)$ не зависит от $a$, а затем покажем, что матрица $S(a)$ может быть выбрана аналитической по $a$.

ЛЕмма 6. При любой изомонодромной деформации собственные значения $\left\{\beta_{i}^{j}\right\}$ матрицы коэфбициентов $B_{i}(a)$ семейства (3.1) и элементы $\left\{\lambda_{i}^{j}\right\}$ матрицы $\Lambda_{i}$ из (3.7) не меняются.

ДокАЗАТЕЛьСтво. Из (3.1), (3.7) следует, что

$$
B_{i}=\lim _{z \rightarrow a_{i}}\left(\left(z-a_{i}\right) \frac{d Y_{i}}{d z} Y_{i}^{-1}\right)=U_{i}\left(a_{i}, a\right)\left(\Lambda_{i}(a)+E_{i}^{0}\right) U_{i}^{-1}\left(a_{i}, a\right),
$$

где $E_{i}^{0}=\lim _{z \rightarrow a_{i}} L_{i}, L_{i}=\left(z-a_{i}\right)^{\Lambda_{i}(a)} E_{i}\left(z-a_{i}\right)^{-\Lambda_{i}(a)}$ голоморфна в $a_{i}$, потому что $\left(z-a_{i}\right) \frac{d Y_{i}}{d z} Y_{i}^{-1}$ голоморфна, а $U_{i}$ голоморфно обратима в $a_{i}$.

Так как матрицы $\Lambda_{i}(a)$ и $E_{i}^{0}$ перестановочны, то из соотношения (3.9) следует, что собственные значения матрицы $B_{i}$ совпадают с собственными значениями $\beta_{i}^{j}=\lambda_{i}^{j}(a)+\rho_{i}^{j}$ матрицы $\Lambda_{i}+E_{i}^{0}$.

По определению при изомонодромной деформации числа $\rho_{i}^{j}$ (логарифмы собственных значений матриц монодромии, нормализованные согласно (1.1)) не меняются. Но, с другой стороны, из непрерывности матриц $B_{i}(a)$ и их собственных значений по параметру а следует, что целые числа $\lambda_{i}^{j}(a)=\beta_{i}^{j}-\rho_{i}^{j}$ также не зависят от $a$. Следовательно, и числа $\beta_{i}^{j}$ также не зависят от $a$. Лемма доказана.

По построению матрица $Y(z, a)$ имеет вид

$$
Y(z, a)=M(z, a)\left(z-a_{i}\right)^{E_{i}}
$$

в некоторой окрестности гиперплоскости $P=\left\{z-a_{i}=0\right\}$, где $M(z, a)$ - мероморфная в окрестности $P$ матричная функция,

\footnotetext{
${ }^{44}$ Гантмахер Ф. Р. Теория матриц. М.: Наука, 1988.
} 
голоморфная вне $P$. Из леммы 6 следует, что для любых $a \in D$ имеет место соотношение

$$
\begin{gathered}
Y(z, a) S(a)=M(z, a) S(a)\left(z-a_{i}\right)^{S^{-1} E_{i} S}, \\
M(z, a) S(a)=U(z, a)\left(z-a_{i}\right)^{\Lambda_{i}} .
\end{gathered}
$$

Нам нужно доказать, что матрицу $S(a)$ можно выбрать аналитической по $a$. Доказательство опирается на следующую лемму.

Лемма 7. Рассмотрим мероморфную в $O \times D\left(a^{0}\right)$ матричу $M(z, a)$ размера $(q, p), q \geqslant p$, голоморфную в $\dot{O} \times D\left(a^{0}\right) \quad$ u имеющую там ранг $p$, где $O \subset \mathbb{C}-$ некоторая окрестность нуля $u \dot{O}=O \backslash\{0\}$. Пусть $A-$ некоторая постоянная диагональная целочисленная матрица. Если для любого значения а из окрестности $\widetilde{U}$ точки $a^{*} \in D$ существуют невырожденная матрица $S(a)$ и голоморфная матрица $U(z, a)$ ранга р в $O \times \widetilde{U}$ такие, что

$$
M(z, a) S(a)=U(z, a) z^{A},
$$

то матричу $S(a)$ можно выбрать аналитической по а в некоторой окрестности $a^{*}$.

ДоказАТЕЛЬСтво. Рассмотрим индукцию по числу $p$ столбцов матрицы $M$.

При $p=1$ доказывать нечего. Предположим, что утверждение верно для всех матриц с числом столбцов, меньшим чем $k$, где $k<p$. Докажем его для $k=p$. Можно считать, что $A=$ $\operatorname{diag}\left(s_{1}, \ldots, s_{p}\right)$, где $s_{1} \geqslant \cdots \geqslant s_{p}, s_{p}=0$. (Этого всегда можно добиться умножением $M$ на скалярную матрицу $z^{-s_{p}} I$.) Пусть в разложении (3.12) $s_{l} \neq 0, s_{l+1}=\cdots=s_{p}=0$. Не ограничивая общности, можно считать также, что $S\left(a^{*}\right)=I$. В этом случае из (3.12) следует, что первые $l$ столбцов матрицы $M\left(0, a^{*}\right)$ нулевые и что ранг $M(0, a)$ равен $p-l$ для всех $a \in \widetilde{U}$. Рассмотрим базисный минор матрицы $M\left(0, a^{*}\right)$. Этот минор включает лишь элементы столбцов с номерами $l+1, \ldots, p$, и в силу его непрерывности соответствующие элементы матрицы $M(0, a)$ образуют ненулевой минор при всех $a \in U^{*}$, где $U^{*} \subset \widetilde{U}-$ некоторая окрестность точки $a^{*}$. Так как ранг матрицы $M(0, a)$ равен $p-l$ для всех $a$, то соответствующий минор является базисным минором для $M(0, a)$ при всех $a \in U^{*}$. Поэтому каждый $j$-й 
столбец $M(0, a)$ с номером $0<j<l+1$ выражается единственным образом в виде линейной комбинации столбцов с номерами $l+1, \ldots, p$ и коэффициенты $c_{k}^{j}$ каждой такой линейной комбинации являются голоморфными функциями в $U^{*}$. Рассмотрим нижнетреугольную матрицу $R_{1}(a)$ с единичными элементами на главной диагонали и такую, что ее $j$-й столбец совпадает со столбцом $\left(0, \ldots, 0,1,0, \ldots, 0,-c_{l+1}^{j}, \ldots,-c_{p}^{j}\right)^{t}$ при $j<l+1$, а столбцы с номерами $j$, большими чем $l$, совпадают со столбцами $e_{j}$ стандартного базиса пространства $\mathbb{C}^{p}$. Построенная матрица голоморфна при всех $a \in U^{*}$, и согласно построению

$$
M(z, a) R_{1}(a)=M^{1}(z, a) z^{A^{1}},
$$

где $A^{1}=\operatorname{diag}\left(s_{1}^{1}, \ldots, s_{p}^{1}\right), s_{l+1}^{1}=\cdots=s_{p}^{1}=0$. Можно считать, что $s_{1}^{1} \geqslant s_{2}^{1} \geqslant \cdots \geqslant s_{l}^{1}$. (Этого всегда можно добиться умножением $R_{1}$ на подходящую постоянную блочно-диагональную матрицу $T=$ $\operatorname{diag}\left(T^{\prime}, I\right)$ с блоком $T^{\prime}$ размера $\left.l.\right)$

Из (3.12), (3.13) следует, что

$$
M^{1}(z, a) z^{A^{1}} S^{1}(a)=U(z, a) z^{A},
$$

где $S^{1}(a)=R_{1}^{-1}(a) S^{-1}(a)$ имеет верхнетреугольный блочный вид $S^{1}=\left(\begin{array}{cc}S_{1} & * \\ 0 & S^{\prime}\end{array}\right)$, потому что лишь последние $p-l$ столбцов матриц $M^{1}(z, a) z^{A^{1}}$ и $U(z, a) z^{A}$ не обращаются в нуль при $z=0$.

Рассмотрим матрицы $M_{1}(z, a), U_{1}(z, a)$, образованные первыми $l$ столбцами матриц $M^{1}(z, a) z^{A^{1}}, U(z, a)$ соответственно и соответствующий блок $A_{1}$ матрицы $A$. В этом случае получаем, что для каждого $a \in U^{*}$

$$
M_{1}(z, a) S_{1}(a)=U_{1} z^{A_{1}} .
$$

Так как $l<p$, из предположения индукции для $l$ следует, что матрицу $S_{1}(a)$ можно выбрать голоморфной в некоторой окрестности $U^{\prime} \subset U^{*}$ точки $a^{*}$. Рассмотрим матрицу $R_{2}=\operatorname{diag}\left(S_{1}, I\right)$. Матрица $R(a)=R_{1}(a) R_{2}(a)$ голоморфно обратима в $U^{\prime}$, и по построению

$$
M(z, a) R(a)=M^{1}(a) z^{A^{1}} R_{2}(a)=V(z, a) z^{A}
$$

для некоторой голоморфной матричной функции $V(z, a)$ ранга $p$ в $O \times U^{\prime}$. 
Из соотношения (3.7) следует, что матрица $S(a)$ имеет ту же блочно-диагональную форму, что и матрицы $\Lambda_{i}, E_{i}$. Следовательно, мы можем ограничиться доказательством теоремы для каждого блока матриц $\Lambda_{i}, E_{i}, S(a)$ и для матриц $M_{j}(z, a)$, образованных соответствующими столбцами $M$ в отдельности. Теперь локальный вариант теоремы для каждого такого набора матриц следует из леммы 7 (после замены $z \mapsto z-a_{i}$ независимой переменной).

Доказательство в глобальном варианте представлено в [9].

Напомним, что матрица $B_{i}$ коэффициентов фуксовой системы называется резонансной, если для какой-либо пары ее собственных значений разность между этими собственными значениями является натуральным числом. Наибольшее число $r_{i}$ из всех таких разностей называется максимальным $і$-резонансом системы.

Согласно (3.7) и $(3.9)$

$$
r_{i}=\max _{j=1, \ldots, k}\left({ }^{1} \lambda_{1}^{j}-{ }^{s_{j}} \lambda_{i}^{j}\right) .
$$

Следующая теорема дает описание общего вида формы $\omega$ из теоремы 22 .

Теорема 24. Любая матричная дифференциальная 1-форма $\omega$ на

$$
\overline{\mathbb{C}} \times D\left(a^{0}\right) \backslash \bigcup_{i=1}^{n}\left\{z-a_{i}=0\right\},
$$

задающая изомонодромную деформацию (3.1) (см. теорему 22) имеет вид

$$
\omega=\sum_{i=1}^{n} \frac{B_{i}(a)}{z-a_{i}} d\left(z-a_{i}\right)+\sum_{l=1}^{n} \sum_{k=1}^{n} \sum_{t=1}^{r_{l}} \frac{\gamma_{t, k, l}(a)}{\left(z-a_{l}\right)^{t}} d a_{k}+\sum_{r=1}^{n} \gamma_{r}(a) d a_{r},
$$

где $\gamma_{t, k, l}(a), \gamma_{r}(a)$ голоморфны в $D\left(a^{0}\right), r_{l}$ - максимальный l-резонанс системы (3.1) при $а=a_{0}$.

ДокАЗАТЕЛЬСтво. По теореме 23 произвольная изомонодромная фундаментальная матрица $Y(z, a)$ семейства (3.1) после умножения на некоторую невырожденную постоянную матрицу $T$ 
имеет следующее разложение в окрестности гиперплоскости $P=$ $\left\{z-a_{i}=0\right\}$ :

$$
Y(z, a) T=U_{i}(z, a)\left(z-a_{i}\right)^{\Lambda_{i}} S^{-1}(a)\left(z-a_{i}\right)^{E_{i}}
$$

для некоторых голоморфно обратимых матриц $U_{i}, S(a)$. Поэтому в окрестности $P$

$$
\omega=d Y Y^{-1}=\omega_{1}+\omega_{2},
$$

где

$$
\begin{aligned}
\omega_{1}= & d U_{i} U_{i}^{-1} \\
& +\frac{U_{i}}{z-a_{i}}\left(\Lambda_{i}+\left(z-a_{i}\right)^{\Lambda_{i}} S_{i}^{-1} E_{i} S_{i}\left(z-a_{i}\right)^{-\Lambda_{i}}\right) U_{i}^{-1} d\left(z-a_{i}\right) \\
= & \frac{B_{i}}{z-a_{i}} d\left(z-a_{i}\right)+\text { некоторая голоморфная форма, }
\end{aligned}
$$

так как по условию теоремы $\omega$ имеет полюс первого порядка в точке $a_{i}$ для любого фиксированного $a_{i}$. Заметим, что форма

$$
\omega_{2}=U_{i}\left(z-a_{i}\right)^{\Lambda_{i}} d_{a} S_{i}^{-1} S_{i}\left(z-a_{i}\right)^{-\Lambda_{i}} U_{i}^{-1}
$$

имеет полюс порядка не больше чем $r_{i}$ на $P$ в силу блочно-диагональной структуры матриц $\Lambda_{i}$ и $S_{i}$ и определения (3.14) максимального $i$-резонанса $r_{i}$ системы.

СЛЕДСТВИЕ 4. Если форма $\omega$ определяет изомонодромную деформачию (3.1), то пфаффова система (3.2) с формой коэффициентов $\omega$ имеет регулярные особые точки на дивизоре $\bigcup_{i=1}^{n}\left\{z-a_{i}=0\right\}$.

В этом смысле все изомонодромные деформации фуксовых систем являются регулярными деформациями.

СЛЕДСТВИЕ 5. Если для каждой матрицы монодромии $G_{i}$ семейства (3.1) и для любого $\lambda \in \mathbb{C}$ имеет место неравенство

$$
\operatorname{rank}\left(G_{i}-\lambda I\right) \geqslant p-1
$$

то изомонодромная деформация (3.1) задается дифберенциальной формой $\omega$ вида (3.6). 
ДокАЗАТЕльСтво. Условие следствия означает, что любую изомонодромную фундаментальную матрицу умножением на некоторую постоянную невырожденную матрицу $T_{i}$ можно привести к такому виду, что каждый блок $G_{i}^{j}$ ее матрицы монодромии $G_{i}$ будет жордановой клеткой. В [5] показано, что в этом случае матрицу $S(a)$ в теореме 23 можно выбрать равной единичной матрице $I$. Следствие доказано.

СлЕДСТвиЕ 6. Если при любом $i$ матрица $B_{i}$ семейства (3.1) не имеет резонансов, то любая форма $\omega$, задающая изомонодромную деформачию (3.1), имеет вид (3.6).

ДокАзАТЕЛЬСтво. В рассматриваемом случае $r_{i}=0$ для любого $i$.

В следующем примере приведена изомонодромная деформация, которая не сводится к деформации Шлезингера (ни к нормализованной, ни к ненормализованной).

ПРимеР 3. Семейство

$$
\begin{aligned}
\frac{d y}{d z}=( & \left(\begin{array}{cc}
1 & 0 \\
-\frac{2 a}{a^{2}-1} & 0
\end{array}\right) \frac{1}{z+a}+\left(\begin{array}{ll}
0 & -6 a \\
0 & -1
\end{array}\right) \frac{1}{z} \\
& +\left(\begin{array}{cc}
2 & 3+3 a \\
\frac{1}{1+a} & -1
\end{array}\right) \frac{1}{z-1} \\
& \left.+\left(\begin{array}{cc}
-3 & -3+3 a \\
\frac{1}{a-1} & 2
\end{array}\right) \frac{1}{z+1}\right) y
\end{aligned}
$$

фуксовых систем является изомонодромным и задается следующей вполне интегрируемой дифференциальной формой:

$$
\begin{aligned}
& \omega=\left(\begin{array}{cc}
1 & 0 \\
-\frac{2 a}{a^{2}-1} & 0
\end{array}\right) \frac{d(z+a)}{z+a}+\left(\begin{array}{cc}
0 & -6 a \\
0 & -1
\end{array}\right) \frac{d z}{z} \\
& +\left(\begin{array}{cc}
2 & 3+3 a \\
\frac{1}{1+a} & -1
\end{array}\right) \frac{d(z-1)}{z-1} \\
& +\left(\begin{array}{cc}
-3 & -3+3 a \\
\frac{1}{a-1} & 2
\end{array}\right) \frac{d(z+1)}{z+1}+\left(\begin{array}{cc}
0 & 0 \\
\frac{2 a}{a^{2}-1} & 0
\end{array}\right) \frac{d a_{1}}{z+a} .
\end{aligned}
$$


Эта форма имеет вид (3.15), и она не сводится к виду (3.6). Так как слагаемое $\sum_{i=1}^{n} \gamma_{i}(a) d a_{i}$ в записи формы отсутствует, этот пример является примером так называемой нормализованной деформации.

Каждая дифференциальная форма $\omega$, задающая изомонодромную деформацию (3.1), имеет вид $\omega=\omega_{s}+\sum_{i=1}^{n} \psi_{i}(z, a) d a_{i}$ (см. (3.15), (3.3)). Верно ли, что слагаемое $\sum_{i=1}^{n} \psi_{i}(z, a) d a_{i}$ определяется однозначно "главной частью" $\omega_{s}$ (мы называем эту часть "главной", потому что она выписывается непосредственно по коэффициентам фуксова семейства)? Ответ на этот вопрос отрицателен.

Имеется следующая свобода в выборе $\sum_{i=1}^{n} \psi_{i}(z, a) d a_{i}$ : можно заменить изомонодромную матрицу $Y(z, a)$, описывающую нашу деформацию, на $Y(z, a) R(a)$, где $R(a)$ принадлежит централизатору матриц монодромии $G_{1}, \ldots, G_{n}$ матрицы $Y(z, a)$. Ясно, что эта замена не меняет форму $\omega_{s}$, но может изменить форму $\sum_{i=1}^{n} \psi_{i} d a_{i}$. При этом имеет место следующее утверждение ${ }^{45}$.

ПРЕДЛОЖЕНИЕ 7. Если монодромия фуксового семейства (3.1) неприводима, то дифференииальная форма $\omega$, задающая изомонодромную деформацию (3.1) (см. теорему 24), определяется однозначно семейством (3.1) с точностъю до слагаемого $d f(a) f^{-1}(a) I$, где $f(a)$ - произвольная голоморфная функиия на $D\left(a^{0}\right)$.

ДокАЗАТЕЛЬСтво. Рассмотрим какую-либо изомонодромную матрицу $Y(z, a)$ семейства (3.1). Из неприводимости монодромии и из леммы Шура следует, что любая другая изомонодромная матрица $Y^{\prime}(z, a)$ с той же монодромией должна иметь вид $Y^{\prime}(z, a)=Y(z, a) R(a)$, где $R(a)=f(a) I$ - некоторая скалярная матрица. Поэтому

$$
\begin{aligned}
\omega^{\prime} & =d Y^{\prime}(z, a)\left(Y^{\prime}\right)^{-1}(z, a) \\
& =d Y(z, a) Y^{-1}(z, a)+Y(z, a) d R(a) R^{-1}(a) Y(z, a) \\
& =\omega+d f(a) f^{-1}(a) I
\end{aligned}
$$

${ }^{45}$ Iwasaki K. et al. From Gauss to Painlevè. A modern theory of special functions. Braunschweig: Vieweg, 1991. 
Если монодромия семейства (3.1) приводима, то оно может иметь нетривиальные симметрии вида

$$
\Gamma(z, a)=Y(z, a) C(a) Y^{-1}(z, a),
$$

где $C(a)$ принадлежит централизатору матриц монодромии матрицы $Y(z, a)$. Это приводит к появлению различных дифференциальных форм, задающих данную деформацию (3.1) (даже в случае, когда набор матриц коэффициентов системы семейства является неприводимым).

\section{Список публикаций}

[1] Болибрух А.А., "Проблема Римана-Гильберта" // УМH, 1990, 45(2), 3-47.

[2] Болибрух А. А., "О построении фуксова дифференциального уравнения по представлению монодромии" // Матем. заметки, 1990, 48(5), 22-34.

[3] Болибрух А.А., "О достаточных условиях положительной разрешимости проблемы Римана-Гильберта" // Матем. заметки, 1992, 51(2), 9-19.

[4] Болибрух А. А., "Фуксовы системы с приводимой монодромией и проблема Римана-Гильберта" / / Нелинейные операторы в глобальном анализе. Новое в глобальном анализе. Изд-во Воронежского ун-та, 1991. 5-20.

[5] Болибрух А.А., "21-ая проблема Гильберта для линейных фуксовых систем" // Трудъ МИАН, 1994, 206, 160.

[6] Болибрух А.А., "Об аналитическом преобразовании к стандартной биркгофовой форме" // Докл. РАН, 1994, 334(5), 553-555.

[7] Болибрух А. А., "Об аналитическом преобразовании к стандартной биркгофовой форме" // Трудъ МИАН, 1994, 203, 33-40.

[8] Болибрух А. А., "К вопросу о существовании фуксовых систем с данными асимптотиками" // Трудъ МИАН, 1997, 216, 32-44. 
[9] Болибрух А.А., "Об изомонодромных слияниях фуксовых особенностей" // Труды МИАН, 1998, 221, 127-142.

[10] Болибрух А. А., "О фуксовых системах с заданными асимптотиками и монодромией" // Трудъ МИАН, 1999, 224, 112-121.

[11] Болибрух А. А., "Мероморфное преобразование к биркгофовой стандартной форме в малых размерностях" // Труды МИАН, 1999, 225, 87-95. 